\title{
An interlaboratory comparison of measurements from filament-stretching rheometers using common test fluids ${ }^{\text {a) }}$
}

\author{
Shelley L. Anna \\ Division of Engineering and Applied Sciences, Harvard University, Cambridge, \\ Massachusetts 02138 \\ Gareth H. McKinley ${ }^{\text {b) }}$ \\ Department of Mechanical Engineering, Massachusetts Institute of Technology, \\ Cambridge, Massachusetts 02139 \\ Duc A. Nguyen and Tam Sridhar \\ Department of Chemical Engineering, Monash University, Clayton, Victoria, \\ Australia \\ Susan J. Muller \\ Department of Chemical Engineering, University of California-Berkeley, \\ and Lawrence Berkeley National Laboratory, Berkeley, California 94720 \\ Jin Huang and David F. James \\ Department of Mechanical and Industrial Engineering, University of Toronto, \\ Toronto M5S 3G8, Canada
}

(Received 14 June 2000; final revision received 9 October 2000)

\section{Synopsis}

Following development of a filament-stretching extensional rheometer at Monash University, similar rheometers have been designed and built in other laboratories. To help validate the basic technique, a collaborative program was undertaken to compare results from several instruments. First, three test fluids prepared at the University of California at Berkeley were characterized in steady and transient shear flows there and at the Massachusetts Institute of Technology (M.I.T.), and then tested in extensional rheometers at M.I.T., Monash and the University of Toronto. Each fluid is a constant-viscosity solution of narrow-molecular-weight-distribution polystyrene dissolved in oligomeric polystyrene. The solute molecular weights are $2.0,6.5$, and $20 \mathrm{million} \mathrm{g} / \mathrm{mol}$, and the polymer concentration in each fluid is $0.05 \mathrm{wt}$. \%. From linear viscoelastic measurements, the Zimm relaxation times of the fluids are found to be 3.7, 31, and $150 \mathrm{~s}$, respectively. The scaling of relaxation times with molecular weight indicates better-than-theta solvent quality, a finding

\footnotetext{
a) This paper was presented by D. F. James at the Society of Rheology Annual Meeting in Madison, Wisconsin, October 1999.

b) Author to whom correspondence should be addressed; electronic mail: gareth@mit.edu
}

(c) 2001 by The Society of Rheology, Inc.

J. Rheol. 45(1), January/February 2001

$0148-6055 / 2001 / 45(1) / 83 / 32 / \$ 20.00$

83 
consistent with independent intrinsic viscometry measurements of equilibrium coil size. Each fluid was tested in the three filament stretching rheometers at similar Deborah numbers. Despite variations in instrument design and the general difficulty of the technique, transient Trouton ratios measured in the three instruments are shown to agree quantitatively. (c) 2001 The Society of Rheology. [DOI: 10.1122/1.1332388]

\section{INTRODUCTION}

The development of a filament stretching apparatus by Sridhar and coworkers has enabled the transient extensional flow behavior of mobile fluids to be characterized, particularly the behavior of dilute polymer solutions [Sridhar et al. (1991)]. Although the importance of extensional rheology measurements has been recognized since the turn of the century [see (Petrie (1979) for a historical account], generating purely extensional flows of mobile fluids has proven to be extremely difficult. Various devices have been developed to measure the "extensional viscosity," $\eta_{E}$, of a mobile fluid, including opposed jet devices, spin-line rheometers, and two- and four-roll mills. These techniques, reviewed in detail elsewhere, all have serious drawbacks, among them an unknown preshear history, "contamination" of the extensional flow field with regions of shearing, and an inability to approach steady state conditions [Gupta and Sridhar (1988); James and Walters (1994)]. An international "round-robin" study in the Journal of Non-Newtonian Fluid Mechanics in 1990 attempted to quantify the differences between many of these devices by comparing measurements for the same fluid, denoted M1 [Sridhar (1990)]. This study showed that different devices can yield widely different results at a given extensional rate. In fact, James and Walters compiled the data from this study and the plot shows over three decades of variation in measurements of $\eta_{E}$ as a function of imposed strain rate $\dot{\varepsilon}$ [James and Walters (1994)]. James and Walters note that the "values of $\eta_{E} \ldots$ are transient values and the disparity makes it clear how strongly these values depend upon variables other than strain rate." The round-robin study of the M1 fluid demonstrated that a new device was needed which could overcome some of the drawbacks of earlier devices and allow systematic study of other dependent variables.

As a part of this 1990 study, a "falling plate" device was introduced by Matta and Tytus [Matta and Tytus (1990)] which would subsequently evolve into the filament stretching apparatus. By simply placing a small amount of fluid between two circular plates and allowing the bottom one to fall under gravity, Matta and Tytus were able to generate a nearly pure extensional flow and to calculate the tension in the fluid filament during stretching. Motivated by this work, Tirtaatmadja and Sridhar designed a device to control the separation of the two endplates such that the fluid is subjected to nearly ideal uniaxial extension while the force on one plate is measured. In this device, the evolution of fluid stress from equilibrium to steady state can be followed [Sridhar et al. (1991); Tirtaatmadja and Sridhar (1993)]. Several groups have subsequently built similar filament stretching devices and reported results for various test fluids [Kröger et al. (1992); Ooi and Sridhar (1993); Berg et al. (1994); Ooi and Sridhar (1994); Solomon and Muller (1996b); Spiegelberg et al. (1996); Spiegelberg and McKinley (1996); van Nieuwkoop and Muller von Czernicki (1996); Jain et al. (1997); Verhoef et al. (1999)]. The flow kinematics, including the effect of the nonideal flow conditions near the rigid endplates, have been explored by researchers, and still others have used data from filament stretching devices to make quantitative comparisons with constitutive models [Shipman et al. (1991); Tirtaatmadja and Sridhar (1995); Gaudet et al. (1996); Kolte et al. (1997); Sizaire and Legat (1997); Szabo (1997); Remmelgas et al. (1998); Yao and McKinley (1998); Olagunju (1999); Yao et al. (2000)]. In addition, the device has been used to 
examine stress relaxation and birefringence in uniaxial extension [Orr and Sridhar (1996); Spiegelberg and McKinley (1996); Doyle et al. (1998); Sridhar et al. (2000)].

The goal of the current study is to investigate the reliability of filament stretching rheometers and to determine their utility as a quantitative method for characterizing the transient extensional rheology of viscoelastic liquids. In the following sections, we compare the transient stress growth in well-characterized test fluids measured in three different filament stretching devices that have been built independently in different laboratories. We highlight important practical considerations and procedures needed to achieve good agreement in results from these different devices.

In Sec. II, we describe the composition and properties of the three test fluids used in this interlaboratory project. Measurements of intrinsic viscosity, steady and dynamic shear rheology, and transient startup and cessation of shear flow are presented. Furthermore, it is found that the fluid response is consistent with a Zimm spectrum of relaxation times, and we demonstrate remarkably good agreement between scaling exponents obtained from intrinsic viscosity measurements and small-amplitude oscillatory shear data. With this complete characterization in shear flows as a foundation, we proceed with measurements of extensional viscosity and compare data from the three different instruments for the three test fluids. In Sec. III, we describe the three filament stretching devices, and compare the fluid response in different ways to show that nearly ideal kinematics are achieved. Finally, in Sec. IV we present measurements of the transient extensional rheology of the three test fluids for a range of imposed strain rates. We hope that this study will provide a guide for experimenters who wish to use filament stretching rheometry as a standard characterization technique.

\section{CHARACTERIZATION OF COMMON TEST FLUIDS}

\section{A. Fluid composition}

The three fluids used in this study, denoted SM-1, SM-2, and SM-3, are dilute polymer solutions with nearly constant shear viscosities, which enable the effects of elasticity to be isolated from those of shear thinning [Boger (1977/78); Boger and Nguyen (1978)]. Each solution has the same concentration, $c=0.05 \mathrm{wt}$. \%, of high molecular weight polystyrene (Pressure Chemical, P.D.I. <1.20) dissolved in oligomeric styrene (Piccolastic A5 Resin, Hercules). The mass-average molecular weights of the polystyrene are $2.0,6.5$, and $20 \times 10^{6} \mathrm{~g} / \mathrm{mol}$, respectively, and the density of each fluid is $1.02 \mathrm{~g} / \mathrm{cm}^{3}$. The manufacturer-reported values of the polydispersity indices for the three solutes are $1.03,1.05$, and 1.20 , respectively. The three fluids were prepared at the University of California at Berkeley. In each case, the high molecular weight polymer was dissolved directly in the oligomeric resin, and the fluid was gently rolled once a day for 2 months to ensure a homogeneous mixture.

The first step of the characterization was measurement of quiescent solution properties, including polymer molecular weight, coil size, and solvent quality, which were determined through a combination of intrinsic viscometry, gel permeation chromatography (GPC), and static light scattering at UC Berkeley. Manufacturer-reported molecular weights of the starting polymers were confirmed by GPC in tetrahydrofuran and by static light scattering in dioctyl phthalate at $22{ }^{\circ} \mathrm{C}$, the theta point for polystyrene. Static light scattering also yielded the radii of gyration of the coils under theta conditions $R_{g, \theta}$; details of these measurement techniques are reported elsewhere [Solomon and Muller (1996a); Lee et al. (1997)]. The measured radii of gyration under theta conditions were in excellent agreement with the values calculated from the molecular weight and the characteristic ratio $C_{\infty}$ as 
TABLE I. Equilibrium properties and molecular weight scaling exponents for SM Boger fluids. The concentration $c$ of all three solutions is fixed at $c=0.00051 \mathrm{~g} / \mathrm{mL}$. The excluded volume exponent is determined to be $\nu=0.52 \pm 0.015$, and the coil expansion exponent, $\gamma=0.02 \pm 0.015$.

\begin{tabular}{cccccccc}
\hline \hline & $\begin{array}{c}M_{w} \\
(\mathrm{~g} / \mathrm{mol})\end{array}$ & $\begin{array}{c}{[\eta]} \\
(\mathrm{mL} / \mathrm{g})\end{array}$ & $\begin{array}{c}R_{g} \\
(\mathrm{~nm})\end{array}$ & \multicolumn{1}{c}{$L$} & $\begin{array}{c}c_{1}^{*} \\
(\mathrm{~g} / \mathrm{mL})\end{array}$ & $\begin{array}{c}c_{2}^{*} \\
(\mathrm{~g} / \mathrm{mL})\end{array}$ & $c / c_{2}^{*}$ \\
\hline SM-1 & $2.0 \times 10^{6}$ & 120 & 41 & 88 & 0.0083 & 0.0011 & 0.44 \\
$\mathrm{SM}-2$ & $6.5 \times 10^{6}$ & 250 & 78 & 164 & 0.0040 & 0.00058 & 0.87 \\
$\mathrm{SM}-3$ & $2.0 \times 10^{7}$ & 430 & 133 & 277 & 0.0023 & 0.00034 & 1.50 \\
\hline \hline
\end{tabular}

$$
R_{g, \theta}^{2}=C_{\infty} n l^{2},
$$

where $C_{\infty}=9.85$ [Brandrup et al. (1999)], $n$ is the number of carbon-carbon bonds in the backbone, and $l=1.54 \AA$ is the length of a carbon-carbon bond [Flory (1953)].

Direct measurements of coil sizes and solvent quality in these solutions via static light scattering could not be made because of insufficient contrast in the scattering between the oligomeric styrene solvent and the high molecular weight polystyrene solute. Instead, these measurements were made through intrinsic viscometry as described below.

For each molecular weight, a series of dilutions were prepared from a 0.05 wt. \% master solution and viscosities were measured in a capillary viscometer or in a Rheometrics RMS-800 mechanical spectrometer using cone-and-plate fixtures. The limiting viscosity number (or intrinsic viscosity) $[\eta]$ is defined through an expansion of the solution viscosity $\eta$ in concentration $c$ as

$$
\eta=\eta_{s}\left(1+[\eta] c+k_{H}[\eta]^{2} c^{2}+\ldots\right),
$$

and was determined by a dual Huggins-Kramer extrapolation of the viscosity data to infinite dilution. In the above equation, $\eta_{s}$ is the (oligomeric styrene) solvent viscosity and $k_{H}$ is the Huggins coefficient. The resulting values of [ $\left.\eta\right]$ are given in Table I, along with other properties of the three solutions. The radius of gyration $R_{g}$ for each fluid was then determined from the Flory-Fox equation:

$$
[\eta]=\Phi_{0} R_{g}^{3} / M_{w},
$$

where $\Phi_{0}$ is a universal constant, equal to $3.67 \times 10^{24} \mathrm{~mole}^{-1}$.

The size of a polymer coil in solution may be related to the solvent quality through either a coil expansion parameter $\alpha$ defined as

$$
\alpha \equiv \frac{R_{g}}{R_{g, \theta}}
$$

or through an excluded volume exponent $\nu$ where

$$
R_{g} \propto M^{\nu}
$$

For a theta solvent, $\alpha$ is unity by definition and $\nu=1 / 2$. For good solvents, $\alpha$ is greater than unity and $0.5<\nu<0.6$.

Alternatively, since $R_{g, \theta} \propto M^{1 / 2}$, one may define a coil expansion exponent $\gamma$ from Eq. (4) above, i.e., $\alpha \propto M^{\gamma}$ where $\gamma=\nu-1 / 2$. The value of the exponent $\gamma$ typically varies from zero for a theta solvent to 0.1 for a good solvent. As shown in Table I, the 
values of $\nu$ and $\gamma$ for our polystyrene-(oligomeric styrene) system were found to be 0.52 \pm 0.015 and $0.02 \pm 0.015$, respectively, indicating that the styrene oligomer is slightly better than a theta solvent.

The above measurements also enable assessment of the diluteness of the three test fluids. Since the polystyrene concentrations are the same in each case, the diluteness decreases with increasing molecular weight. Several measures of diluteness have been proposed in the literature, and a very thorough discussion of these is given by Graessley and by Harrison and co-workers [Graessley (1980); Harrison et al. (1998)]. The most common method of assessing diluteness depends on the magnitude of the intrinsic viscosity. A critical concentration $c_{1}^{*}$ can be defined as

$$
c_{1}^{*}=1 /[\eta]
$$

although Graessley notes that a proportionality factor of 0.77 is more rigorous. The values of $c_{1}^{*}$ given in Table I are well above the concentration of $0.00051 \mathrm{~g} / \mathrm{mL}$ of our solutions, showing that all three fluids lie well within the dilute regime according to this definition. A second, more conservative measure of diluteness is based on the concentration at which the coils at equilibrium begin to physically overlap. This critical concentration $c_{2}^{*}$ is given by

$$
c_{2}^{*}=\frac{M_{w}}{\frac{4}{3} \pi R_{g}^{3} N_{A}},
$$

where $N_{A}$ is Avogadro's number [Graessley (1980)]. By this measure, the SM-3 fluid lies in the semidilute regime, since $c \approx 1.5 c_{2}^{*}$. A plot of the fluid viscosity versus concentration also shows significant curvature at concentrations above $0.034 \mathrm{wt} \%$, indicating that SM-3 is above a nominal value of $c^{*}$, as defined in terms of the relative magnitude of the quadratic term in Eq. (2). However, in this study, SM-3 is considered to be a dilute solution, because we found that the behavior of the fluid in viscometric experiments was consistent with that of a dilute solution.

\section{B. Steady and dynamic shear rheology}

The rheology of the three fluids in both steady and dynamic shear flows was characterized at the Massachusetts Institute of Technology (M.I.T.) using a TA Instruments AR1000N cone-and-plate rheometer. First normal stress differences were also measured using a Rheometric Scientific RMS-800 cone-and-plate rheometer to obtain values of the first normal stress coefficient $\Psi_{1}(\dot{\gamma})$. Controlled shear stress measurements were carried out at three temperatures: 15,25 , and $35^{\circ} \mathrm{C}$, and time-temperature superposition was used to obtain master curves for the steady and dynamic material functions over 6 decades in shear rate. Throughout this text, the official Society of Rheology nomenclature is used for the material functions studied [Dealy (1995)].

The temperature dependence of the viscometric properties was determined by continuously increasing the temperature from 15 to $35^{\circ} \mathrm{C}$ over the period of $1 \mathrm{~h}$ at a constant rate of $(\Delta T / \Delta t)=0.056 \mathrm{~K} / \mathrm{s}$ while keeping the applied shear stress fixed at $10.0 \mathrm{~Pa}$. No hysteresis was observed in subsequent tests with a decreasing temperature ramp. This confirms that evaporation of the viscous oligomeric styrene solvent is negligible over this temperature range and the temperature ramp is slow enough that the process is quasistatic. The measured viscosity curve was then normalized by the viscosity at the reference temperature $T_{0}=25^{\circ} \mathrm{C}$ to obtain the shift factor $a_{T}$ as a function of temperature $T$. The 


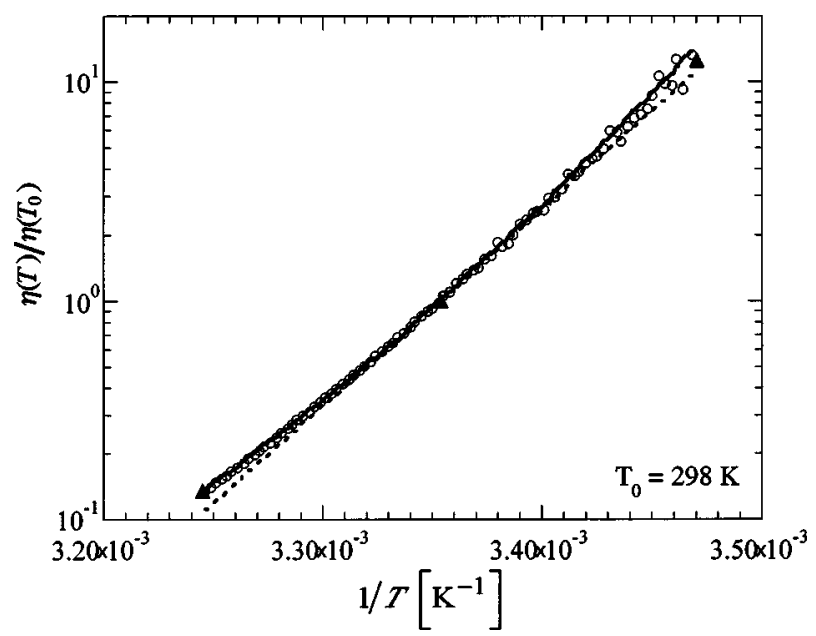

FIG. 1. Temperature dependence of shear viscosity of SM-1 fluid. Experimental points measured in a coneand-plate rheometer $(\bigcirc)$ are compared with manually shifted values $(\boldsymbol{\Delta})$ and fitted curves from Arrhenius and WLF models (dashed and solid lines, respectively).

temperature behavior of the SM fluids is best described by the WLF model, in which the shift factor depends on the temperature according to

$$
a_{T} \equiv \frac{\eta(T)}{\eta\left(T_{0}\right)} \frac{T_{0}}{T}=\frac{\lambda(T)}{\lambda\left(T_{0}\right)}=\exp \left[\frac{-a\left(T-T_{0}\right)}{b+\left(T-T_{0}\right)}\right],
$$

where $a$ and $b$ are constants determined by fitting the equation to experimental data [Bird et al. (1987a)]. In this expression we have dropped the additional factor of $\rho_{0} / \rho(T)$ that appears in the first equality since it is negligibly small over the temperature range of the experiments. Figure 1 shows excellent agreement between the experimentally measured shift factor and the fitted WLF curve for SM-1. The corresponding Arrhenius fit is also shown in order to demonstrate that the Arrhenius function is not strong enough to describe the observed temperature dependence. Both the measured shift factor and the WLF fitted function agree well with values of $a_{T}$ obtained by manually shifting the flow curves measured at 15,25 , and $35^{\circ} \mathrm{C}$ until a smooth master curve was obtained. The best-fit values of $a$ and $b$ for the WLF model are included in Table II. Time-temperature superposition is used to shift both shear and extensional rheology data obtained at a laboratory temperature $T$ to the reference temperature $T_{0}$. Separate tests of the thermal sensitivity of the total shear viscosity $\eta_{0}$ and the solvent viscosity $\eta_{s}$ indicate that each quantity depends on temperature in the same way, and the relationship given in Eq. 8 is used to

TABLE II. Measured viscometric properties and fit parameters for the Zimm bead-spring model for SM fluids. The solvent viscosity, $\eta_{s}=34.0 \mathrm{~Pa} \mathrm{~s}$, and the solvent relaxation time $\lambda_{s}=2.7 \times 10^{-4} \mathrm{~s}$.

\begin{tabular}{cccccccc}
\hline \hline & $\begin{array}{c}M_{w} \\
(\mathrm{~g} / \mathrm{mol})\end{array}$ & $\begin{array}{c}\eta_{0} \\
(\mathrm{Pas})\end{array}$ & $\begin{array}{c}\lambda_{Z} \\
(\mathrm{~s})\end{array}$ & $\begin{array}{c}\Psi_{10} \\
\left(\mathrm{~Pa} \mathrm{~s}^{2}\right.\end{array}$ & $h^{*}$ & $a_{\mathrm{WLF}}$ & $\begin{array}{c}b_{\mathrm{WLF}} \\
(\mathrm{K})\end{array}$ \\
\hline SM-1 & $2.0 \times 10^{6}$ & 39.2 & 3.7 & 20.3 & 0.20 & 16.9 & 75.4 \\
SM-2 & $6.5 \times 10^{6}$ & 46.1 & 31.1 & 428 & 0.15 & 22.4 & 99.6 \\
SM-3 & $2.0 \times 10^{7}$ & 55.5 & 155 & 3510 & 0.18 & 36.9 & 160 \\
\hline \hline
\end{tabular}




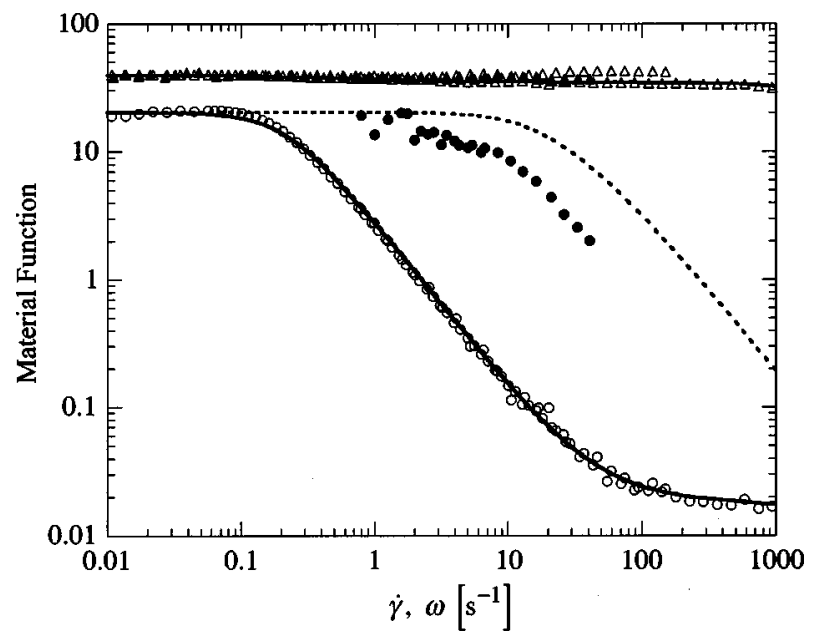

FIG. 2. Steady and dynamic shear rheology of SM-1 fluid. Fitted curves from the Zimm bead-spring model (solid lines) are compared with measurements of the dynamic storage and loss moduli $\left[\eta^{\prime}, \Delta ; 2 \eta^{\prime \prime}(\omega) / \omega, \bigcirc\right]$ obtained in a cone-and-plate rheometer. Predictions from the FENE-P model (dashed lines) are compared with steady shear data $\left[\eta(\dot{\gamma}), \boldsymbol{\Delta} ; \Psi_{1}(\dot{\gamma})\right.$,

shift each of these values. The validity of time-temperature superposition for transient extensional flows of thermorheologically simple fluids has been confirmed by Münstedt and Laun (1979).

Figure 2 shows the steady and dynamic shear material functions for the SM-1 fluid. The SM-2 and SM-3 shear rheology curves are qualitatively similar, with magnitudes of the viscosity $\eta_{0}$ and the first normal stress coefficient $\Psi_{10}$ at zero-shear rate increasing with molecular weight. Each fluid is well described by a Zimm spectrum of relaxation times, as shown by the model curves in Fig. 2. The Zimm model is a bead-spring model in which the polymer chain is approximated as a coarse-grained chain of $N+1$ beads connected by springs of length $Q$. In contrast to the Rouse bead-spring model, this model incorporates hydrodynamic interaction between beads (in a preaveraged sense), and includes both partially free-draining and dominant hydrodynamic interaction limits. In the Zimm model, solvent flow around each bead is affected by the presence of the other beads, and thus internal parts of the chain are hydrodynamically screened. Detailed descriptions of this model and of the corresponding evolution equations for chain conformation are given elsewhere [Bird et al. (1987a); Larson (1988)]. The Zimm spectrum of relaxation times can be obtained from an approximate solution of the eigenvalue problem that results from the coupling between springs. The relaxation time of each mode $i$ is related to the longest relaxation time $\lambda_{Z}$ by the hydrodynamic interaction parameter $h^{*}$ according to the expression

$$
\lambda_{i}=\lambda_{Z} / i^{2+\tilde{\sigma}},
$$

where $\tilde{\sigma} \cong-1.40\left(h^{*}\right)^{0.78}$ [Larson (1988)]. The hydrodynamic interaction parameter $h^{*}$ has a maximum value of 0.25 when the solvent is a theta solvent, and decreases as solvent quality improves and the coils expand.

The dynamic moduli for the Rouse-Zimm bead-spring model may be written compactly in the form 


$$
\begin{gathered}
G^{\prime} \equiv \eta^{\prime \prime} \omega=\frac{c N_{A} k_{B} T}{M_{w}} \sum_{i=1}^{N_{m}}\left\{\frac{\left(\lambda_{Z} \omega\right)^{2}}{\left[i^{2(2+\tilde{\sigma})}+\left(\lambda_{Z} \omega\right)^{2}\right]}\right\}, \\
G^{\prime \prime} \equiv \eta^{\prime} \omega=\eta_{s} \omega+\frac{c N_{A} k_{B} T}{M_{w}} \sum_{i=1}^{N_{m}}\left\{\frac{\left(\lambda_{Z} \omega\right) i^{(2+\tilde{\sigma})}}{\left[i^{2(2+\tilde{\sigma})}+\left(\lambda_{Z} \omega\right)^{2}\right]}\right\},
\end{gathered}
$$

where $k_{B}$ is Boltzmann's constant and $N_{m}$ is the number of modes. In addition, the zero-shear-rate viscometric properties of the dilute polymer solution are described by

$$
\begin{gathered}
\eta_{0}=\eta_{s}+\frac{c N_{A} k_{B} T}{M_{w}} \lambda_{Z} \sum_{i=1}^{N_{m}} \frac{1}{i^{(2+\tilde{\sigma})}}, \\
\Psi_{10}=2 \frac{c N_{A} k_{B} T}{M_{w}} \lambda_{Z}^{2} \sum_{i=1}^{N_{m}} \frac{1}{i^{2(2+\tilde{\sigma})}} .
\end{gathered}
$$

In practice, $\lambda_{Z}$ is obtained by fitting Eqs. (10)-(12) to the measured dynamic moduli and matching the respective zero-shear-rate values. Varying $h^{*}$ allows better agreement between the experimental and fitted curves at intermediate angular frequencies. For the best fit, it was found that a small amount of solvent elasticity was needed in order to incorporate the high frequency behavior observed in both the storage and loss moduli. The value chosen for the solvent relaxation time $\lambda_{s}$ was based on the frequency $\left(\omega^{*}\right.$ $\left.\sim 1 / \lambda_{s}\right)$ at which the solvent elasticity became important. Table II presents the viscometric properties and the fitted Zimm parameters for the three SM fluids.

Like the equilibrium properties, the molecular weight dependence of the viscometric properties can also be used to investigate polymer-solvent interaction. In the Zimm bead-spring model, the parameter $\nu$ defined by Eq. (5) is connected to the hydrodynamic interaction parameter $h^{*}$. To understand how hydrodynamic interaction affects the molecular weight dependence of the longest relaxation time $\lambda_{Z}$ and the polymer contribution to the viscosity $\eta_{p}=\eta_{0}-\eta_{s}$, we first recognize that the flow of solvent around a single bead on the polymer chain affects the flow around all the other beads in such a way that the overall flow can be described by Stokes flow around a single sphere of radius $\sqrt{6} R_{g}$. In Stokes flow, the drag on a sphere is given by $\mathbf{f}=6 \pi \eta_{s}\left(\sqrt{6} R_{g}\right) \boldsymbol{\nu}$. The relaxation time of the fluid is a function of the diffusivity $D$ of the polymer chain $\lambda_{Z} \sim R_{g}^{2} / D$, and this diffusivity is inversely proportional to the drag in accordance with the Stokes-Einstein relation. Combining these relationships and using Eq. (5), the relaxation time is found to depend on the molecular weight in the following way:

$$
\lambda_{Z} \sim \frac{6 \pi \eta_{s}}{k_{B} T} R_{g}^{3} \sim M_{w}^{3 \nu} .
$$

Substituting this dependence into Eq. (11), we find that the polymer contribution to the viscosity has the following dependence on molecular weight:

$$
\eta_{p} \sim \frac{c N_{A} k_{b} T \lambda_{Z}}{M_{w}} \sim M_{w}^{3 \nu-1} .
$$

Further details of this analysis can be found elsewhere [Bird et al. (1987b); Larson (1988)].

We performed a linear regression technique to plots of the measured values of $\lambda_{Z}\left(M_{w}\right)$ and $\eta_{p}\left(M_{w}\right)=\eta_{0}\left(M_{w}\right)-\eta_{s}$ as functions of molecular weight in order to 


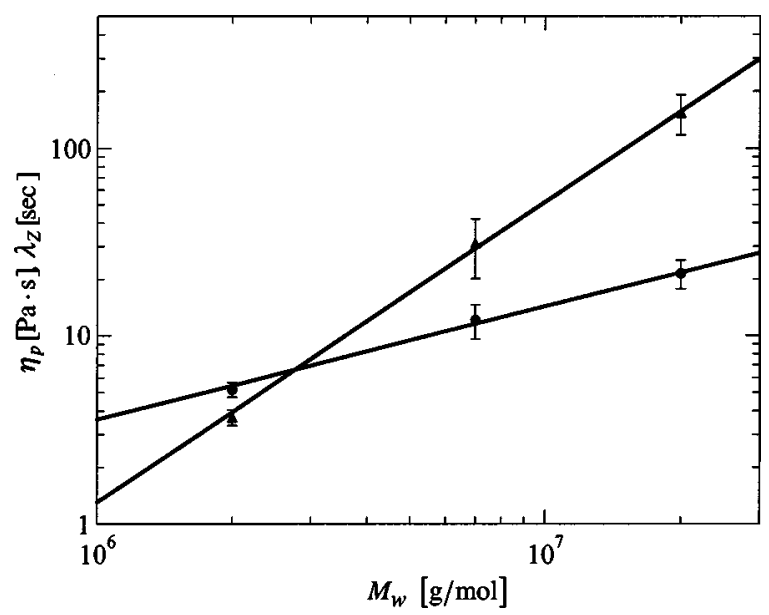

FIG. 3. Molecular weight scaling of the longest relaxation time $\lambda_{Z}(\mathbf{\Delta})$, and of the polymeric contribution to total viscosity $\eta_{p}(\bullet)$ for the three SM fluids. Linear regression was used to obtain a value of 0.54 for the scaling exponent $\nu$, a value appropriate for a solution of nonfree-draining bead spring chains.

determine the value of $\nu$. The values of $\lambda_{Z}$ and $\eta_{p}$ at the reference temperature of $T_{0}=25.0^{\circ} \mathrm{C}$, are shown in Fig. 3, together with error bars and the corresponding linear regression lines. From these lines, the molecular weight dependence of each parameter is found to be

$$
\begin{array}{rlr}
\lambda_{Z}=(2.3 \pm 1.7) \times 10^{-10} M_{w}^{(1.62 \pm 0.05)}, & R^{2}=0.999, \\
\eta_{p}=(6.8 \pm 3.9) \times 10^{-4} M_{w}^{(0.62 \pm 0.04)}, & R^{2}=0.997,
\end{array}
$$

yielding $\nu=0.54 \pm 0.02$ to be consistent with Eqs. (13) and (14). Finally, we note that this value of $\nu$ agrees very well with the value of $0.52 \pm 0.015$ obtained from the intrinsic viscosity measurements, further supporting the idea that the styrene resin acts as a slightly better than theta solvent for the high-molecular-weight polystyrene.

Although the Zimm bead-spring model can describe the rheological behavior of dilute polymer solutions in the linear viscoelastic regime, it cannot describe the behavior when the fluid is subjected to larger deformations. In fact, the Zimm model predicts that the shear viscosity $\eta(\dot{\gamma})$ and the first normal stress coefficient $\Psi_{1}(\dot{\gamma})$ are both constant, independent of shear rate. Figure 2 shows that $\Psi_{1}(\dot{\gamma})$ is in fact far from constant. For the large deformations which the present fluids experience in both shear and extension, the model needs to incorporate nonlinear elasticity. Hence we examine predictions of two bead-spring models, the FENE-P dumbbell and the FENE-PM chain. The FENE-P model, the simpler of the two, is described in detail in Bird et al. (1987b). This model approximates the polymer chain as a single nonlinear spring connecting two beads, and accounts for the finite length of the polymer by limiting the extension of the dumbbell. An additional spring parameter, the finite extensibility $L$, determines the maximum length of the dumbbell. While $L$ can be used as an adjustable parameter, a more physical approach is to require that $L$ be defined by molecular arguments. Following this approach, the finite extensibility is related to the ratio of the maximum contour length of the polymer chain to its equilibrium radius of gyration, as given by 


$$
L^{2}=3\left(\frac{r_{\max }}{R_{g}}\right)^{2} .
$$

Values of $L$ for the SM fluids are given in Table I.

The evolution equations for stresses in a FENE fluid can be derived by balancing the molecular-level forces on each of the dumbbells and taking appropriate ensemble averages to obtain the macroscopic material response [Bird et al. (1987b)]. As shown by Bird et al. (1987b), a closed-form FENE-P model is obtained by making the well-known Peterlin approximation, leading to the following evolution equations:

$$
\begin{aligned}
\lambda_{1} \mathbf{A}_{(1)} & =\mathbf{I}-f(\operatorname{tr} \mathbf{A}) \mathbf{A} \\
f(\operatorname{tr} \mathbf{A}) & =\left[\frac{1}{1-\operatorname{tr} \mathbf{A} / L^{2}}\right],
\end{aligned}
$$

where $\mathbf{A}=\langle\underline{\mathbf{R R}}\rangle$ is the dimensionless configuration tensor describing the ensemble average of the dumbbell orientations. The stresses in the FENE-P fluid are then given by

$$
\begin{gathered}
\boldsymbol{\tau}_{p}=\frac{\eta_{p}}{\lambda_{1}}[f(\operatorname{tr} \mathbf{A}) \mathbf{A}-\mathbf{I}] \\
\boldsymbol{\tau}=\boldsymbol{\tau}_{p}+\boldsymbol{\tau}_{s},
\end{gathered}
$$

where $\tau_{p}$ is the polymeric contribution to the stress and $\tau_{s}$ is the solvent contribution given by $\tau_{s}=\eta_{s} \dot{\gamma}$. The values of the longest relaxation time $\lambda_{1}$ and the polymeric viscosity $\eta_{p}$ in this model are taken from the viscometric properties given in Table II. In the present study, Eqs. (17) and (18) were used to calculate the shear and normal stresses for the simple case of steady shear flow, from which the first normal stress coefficient $\Psi_{1}(\dot{\gamma})$ was computed. The resulting prediction is the dashed curve in Fig. 2, which describes the shear-thinning behavior of $\Psi_{1}(\dot{\gamma})$ qualitatively, but not quantitatively.

The transient rheological response of a dilute polymer solution is more complex than can be captured by a simple closed-form dumbbell model, as shown by recent Brownian dynamics simulations of Kramers chains and bead-spring chains [Doyle and Shaqfeh (1998); Doyle et al. (1998); Hur et al. (2000); Li et al. (2000)]. This complex response is a result of coupling between the flow and the internal degrees of freedom of the chain. Detailed comparisons between simulations and filament stretching experiments have been presented elsewhere [Doyle et al. (1998); Li et al. (2000)] and it is not the purpose of the present work to consider all such comparisons. For simplicity, we instead select the simplest multimode bead-spring chain, namely the FENE-PM model of Wedgewood et al. (1991), which is capable of capturing a spectrum of internal degrees of freedom together with an overall finite extensibility. In this model, $N_{m}$ beads and $N_{m}-1$ nonlinear springs are connected to form a linear bead-spring chain. In order to obtain a closed-form constitutive equation, the connector force in each individual link is replaced with the mean connector force in the $N_{m}$ links. The resulting constitutive equations are given by

$$
\begin{gathered}
\mathbf{A}_{k(1)}=\frac{1}{\lambda_{k}}\left(\mathbf{I}-Z \mathbf{A}_{k}\right), \\
Z=\left[1-\left\{\sum_{k=1}^{N_{m}} \operatorname{tr} \mathbf{A}_{k} / b N_{m}\right\}\right]^{-1},
\end{gathered}
$$


where $\mathbf{I}$ is the identity matrix and $b$ is related to the finite extensibility by $b N_{m}=L^{2}$. The Zimm spectrum of relaxation times found from dynamic shear data are used as the relaxation times $\lambda_{k}$ and the fluid stresses are computed from Eq. (19):

$$
\begin{gathered}
\boldsymbol{\tau}_{k}=n k_{B} T\left(Z \mathbf{A}_{k}-\mathbf{I}\right) \\
\boldsymbol{\tau}=\boldsymbol{\tau}_{s}+\sum_{k=1}^{N_{m}} \boldsymbol{\tau}_{k} .
\end{gathered}
$$

The number of relaxation modes $N_{m}$ can be varied to obtain better fits to the linear viscoelastic data and to the steady and transient shear measurements. We recognize in advance, however, that the FENE-PM model is not capable of capturing all of the dynamics of a Kramers chain. In particular, it does not produce the stress-orientation hysteresis encountered in strong transient shear and extensional flows [Kwan and Shaqfeh (1998); Doyle et al. (1998); Sridhar et al. (2000)]. However, it predicts stresses more accurately than a single dumbbell model, and thus indicates the extent to which the shear and extensional rheology of dilute elastic polymer solutions can be captured by closedform constitutive models. In the next section, we compare predictions from the FENE-PM model to stress growth and relaxation curves measured in stepped shear flows for the SM-2 fluid. In Sec. IV, we compare FENE-PM predictions of extensional viscosity to measurements from the filament stretching rheometers at M.I.T., Monash University, and the University of Toronto.

\section{Transient shear flows}

Transient shear flow experiments were performed at UC Berkeley for all three fluids, using a Rheometric Scientific RMS-800 cone-and-plate rheometer. In this work, a step increase in shear rate was imposed for a prescribed amount of time, and then the rotation of the cone was stopped abruptly. The growth of shear and normal stresses were monitored, along with their relaxation after cessation of shearing. The temperature of all experiments was held constant at $T_{0}=25^{\circ} \mathrm{C}$.

Transient curves for the SM-2 fluid, showing both growth and relaxation of the viscosity and the first normal stress coefficient, are shown in Figs. 4(a) and 4(b). Data for three imposed shear rates, $\dot{\gamma}_{0}=1.0,4.0,8.0 \mathrm{~s}^{-1}$, are shown. As the shear rate increases, the steady state value of viscosity remains essentially constant, while $\Psi_{1}^{+}\left(\dot{\gamma}_{0}, t \rightarrow \infty\right)$ decreases, in agreement with the data in Fig. 2. Prior to reaching a steady state value, the first normal stress coefficient experiences an overshoot, the magnitude of which increases as the shear rate increases. Transient shear curves predicted from the FENE-PM model are shown for each shear rate. Although the steady state values of $\Psi_{1}(\dot{\gamma})$ are overpredicted, both the transient stress overshoot and the shearthinning effect are qualitatively captured.

The relaxation of the shear stress and first normal stress difference following cessation of steady shear flow is also shown in Fig. 4. The relaxation data show that, as the shear rate increases, the rate of decay increases. Predictions from the FENE-PM model also show this trend, although the stress decays much faster than the model indicates. In Fig. 4(a), the FENE-PM model accurately predicts the immediate decade drop in viscosity and is qualitatively correct afterward. In Fig. 4(b), the agreement between experiment and model is qualitative at best. The inability of the FENE-PM model to quantitatively predict 

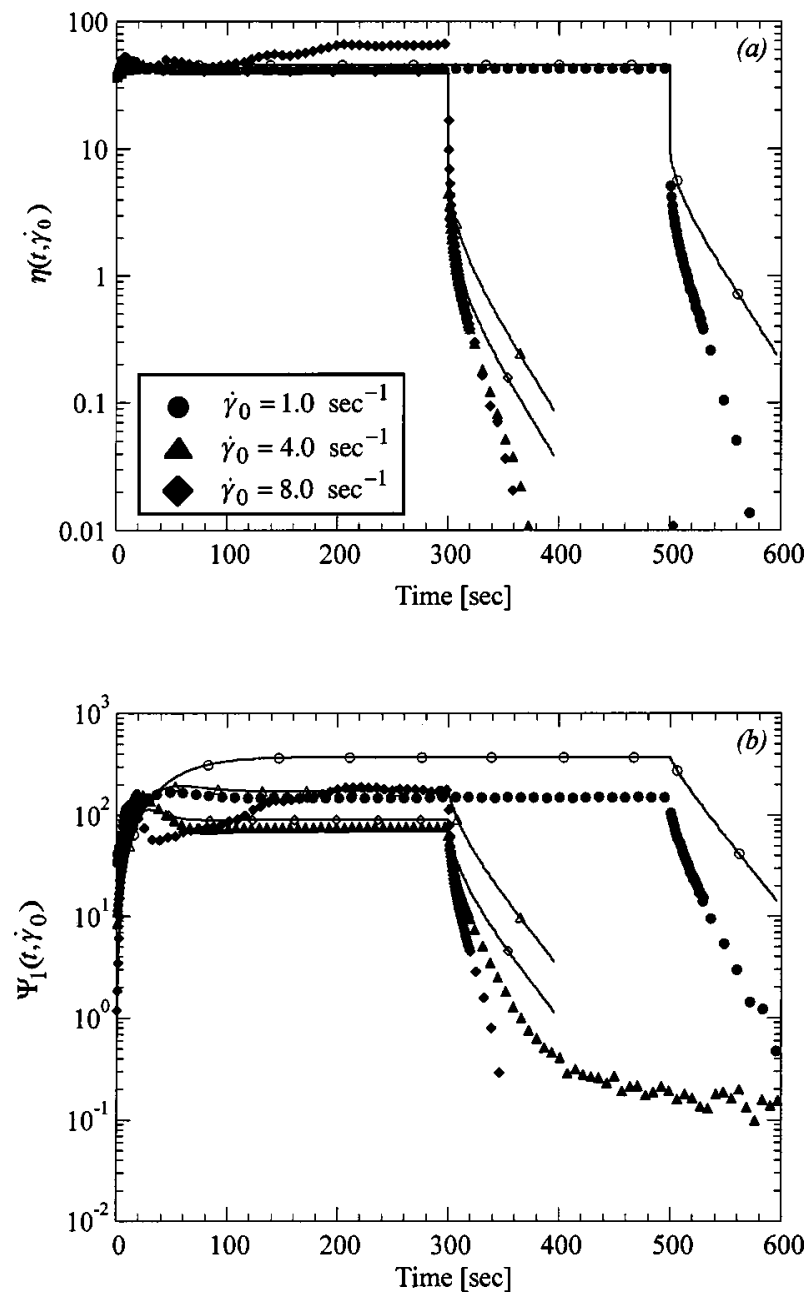

FIG. 4. Startup and cessation of shear flow for SM-2 fluid for three imposed shear rates;

$\dot{\gamma}_{0}=1.0 \mathrm{~s}^{-1},(\boldsymbol{\Delta}) 4.0 \mathrm{~s}^{-1},(\diamond) 8.0 \mathrm{~s}^{-1}$. Predicted curves from the FENE-PM model (solid lines) are compared with measured transient curves (symbols) of (a) viscosity and (b) first normal stress difference.

either the shear-rate dependence of the viscometric properties or the stress relaxation curves arises in part because of the coupling-mode averaging which simplifies computations with the model.

At the highest shear rate $\dot{\gamma}=8.0 \mathrm{~s}^{-1}$ both the shear stress and the first normal stress difference increase erratically with time. This behavior is characteristic of an elastic instability, first observed by Jackson et al. (1984) and later studied in detail by numerous authors [Magda and Larson (1988); McKinley et al. (1991); Byars et al. (1994); MacDonald and Muller (1997)]. This instability was observed in the two most elastic fluids (SM-2 and SM-3) during steady rate sweeps.

\section{INTERLABORATORY COMPARISON OF FILAMENT STRETCHING DATA}

Having fully characterized the linear viscoelastic, steady, and transient shear responses of the test fluids, we now proceed to measure the transient extensional stress growth in three filament stretching devices. 


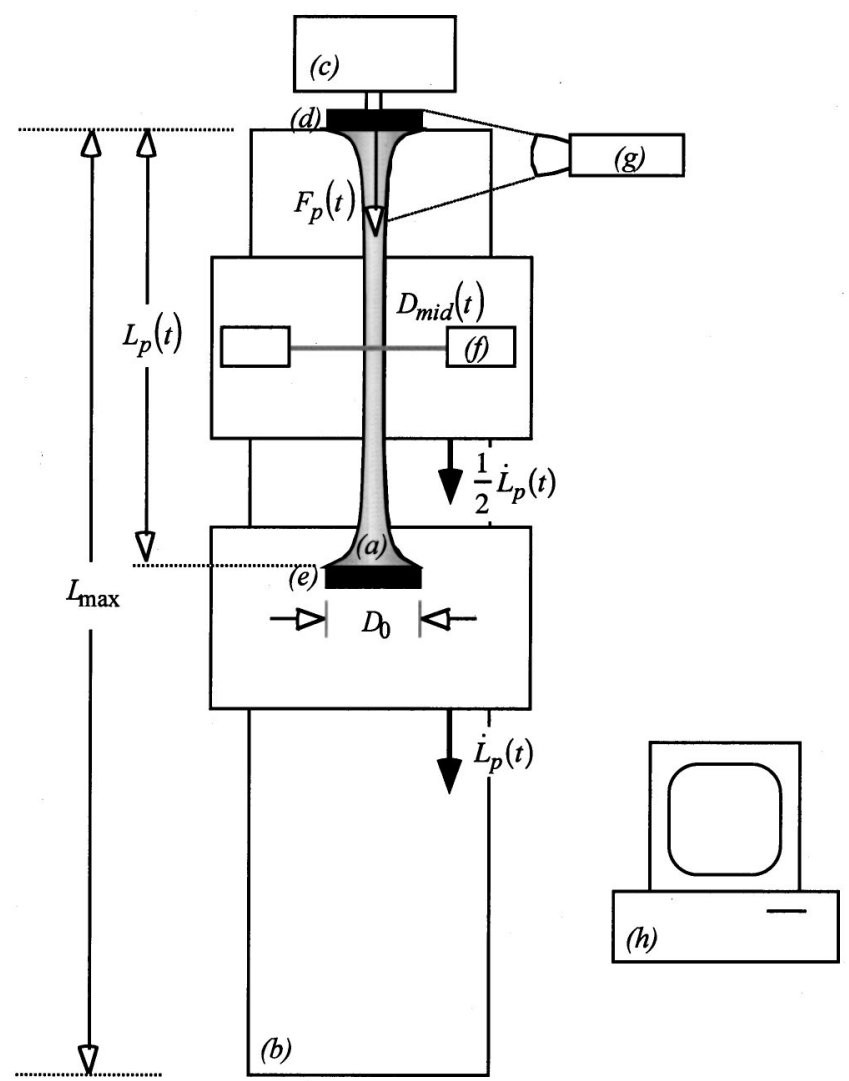

FIG. 5. Schematic diagram of a generic filament stretching device. Basic elements include: (a) fluid filament, (b) linear motor with one or two moving platens, (c) force transducer, (d) top endplate, (e) bottom endplate, (f) diameter sensor, (g) (optional) camera for observing profile near endplate, and (h) computer system for control and data acquisition. Components can be arranged in several different configurations; the specific configurations of the three devices used in this study are summarized in Table III.

\section{A. Filament stretching devices}

The basic elements of a filament stretching device are shown in Fig. 5. An initially cylindrical fluid filament is elongated between two circular endplates, which are attached to a motion control system consisting of either one or two moving platens. Several possible arrangements of the endplates, motor platens, and sensors allow simultaneous measurement of the tensile force in the filament $F_{p}$ and the mid-filament diameter $D_{\text {mid }}$ as the endplates move apart according to a carefully controlled separation history $L_{p}(t)$. Additional important components of the instrument include the data acquisition and control software on an attached computer, a temperature sensor, and possibly equipment to image the flow near the rigid endplate.

In an ideal uniaxial extensional flow, in which the extensional rate is constant, the axial velocity in the fluid $v_{z}$ is proportional to the axial position $z$, leading to an endplate separation profile which increases exponentially with time

$$
L_{p}(t)=L_{0} \exp (\dot{E} t)
$$


TABLE III. Component specifications of the filament stretching devices.

\begin{tabular}{|c|c|c|c|}
\hline & Toronto & M.I.T. & Monash \\
\hline \multicolumn{4}{|c|}{ Flow kinematics and motion control } \\
\hline Motor & $\begin{array}{c}\text { Belt-driven dc } \\
\text { Servo }\end{array}$ & $\begin{array}{c}\text { Linear dc } \\
\text { Servo }\end{array}$ & $\begin{array}{l}\text { Linear dc } \\
\text { Servo }\end{array}$ \\
\hline & top: fixed & top: moves & top: moves \\
\hline Configuration & bottom: moves & bottom: fixed & bottom: fixed \\
\hline$L_{\max }(\mathrm{cm})$ & 200 & 180 & 90/plate \\
\hline$\Delta L_{\min }(\mathrm{cm})$ & - & $1 \times 10^{-4}$ & $1 \times 10^{-4}$ \\
\hline$V_{\max }(\mathrm{cm} / \mathrm{s})$ & 200 & 300 & 200/plate \\
\hline$\Delta V_{\min }(\mathrm{cm} / \mathrm{s})$ & 0.3 & 0.035 & a \\
\hline$\dot{\varepsilon}^{*}(1 / \mathrm{s})$ & 1.50 & 1.67 & 2.22 \\
\hline \multicolumn{4}{|c|}{ Force measurement } \\
\hline Sensor & $\begin{array}{l}\text { Cantilevered } \\
\text { quart beams }\end{array}$ & Strain gage & $\begin{array}{l}\text { Cantilevered } \\
\text { quartz beams }\end{array}$ \\
\hline Configuration & fixed, top & fixed, bottom & moves, bottom \\
\hline$F_{\max }(\mathrm{g} \mathrm{m})$ & 1 or 10 & 10 & 5 \\
\hline$\Delta F_{\min }(\mathrm{g} \mathrm{m})$ & 0.0002 & 0.01 & 0.0001 \\
\hline Sensor response & - & two-pole & - \\
\hline$f_{c}(\mathrm{~Hz})$ & - & 12 & 40 \\
\hline$\gamma_{D}(1 / \mathrm{s})$ & - & 58 & - \\
\hline \multicolumn{4}{|c|}{ Diameter measurement } \\
\hline Sensor & Zumbach ODAC & Omron Z4LA & Zumbach ODAC \\
\hline$D_{\max }(\mathrm{mm})$ & 8 & 10 & 30 \\
\hline$\Delta D_{\min }(\mathrm{mm})$ & 0.05 & 0.01 & 0.05 \\
\hline \multicolumn{4}{|c|}{ Geometry } \\
\hline$D_{0}(\mathrm{~mm})$ & 3.0 & 7.0 & 3.0 \\
\hline$\Lambda_{0}$ & $4 / 3$ & 1.0 & 1.0 \\
\hline \multicolumn{4}{|c|}{ Dimensionless parameters } \\
\hline$\varepsilon_{L}^{\max }$ & 6.90 & 6.24 & 7.09 \\
\hline $\mathrm{De}_{\mathrm{sag}}(\mathrm{SM}-1)$ & 1.43 & 3.33 & 1.43 \\
\hline $\mathrm{De}_{\mathrm{sag}}(\mathrm{SM}-2)$ & 10.2 & 23.7 & 10.2 \\
\hline $\mathrm{De}_{\mathrm{sag}}(\mathrm{SM}-3)$ & 42.1 & 98.3 & 42.1 \\
\hline Bo & 0.75 & 4.1 & 0.75 \\
\hline
\end{tabular}

${ }^{\mathrm{a}}$ Machine reports velocity in steps of $0.25 \mathrm{~cm} / \mathrm{s}$, calculated from length.

where $\dot{E}$ is the imposed constant stretch rate and $L_{0}$ is the initial length. The total deformation of the fluid filament is defined in terms of the Hencky strain, for which the nominal value is computed from the length

$$
\varepsilon_{L}=\ln \left(\frac{L_{p}(t)}{L_{0}}\right)=\dot{E} t
$$

Table III summarizes the specific configuration and geometry details of the three laboratory instruments. The main differences in the devices involve the diameter $D_{0}$ of the rigid endplates and the configuration of the motor platens and force transducers. At M.I.T., $D_{0}=7.0 \mathrm{~mm}$, and the force transducer is mounted to the bottom endplate. At Monash and Toronto, $D_{0}=3.0 \mathrm{~mm}$, and the force transducer is attached to the upper endplate. The three laboratories have different motion configurations: at M.I.T., the top endplate moves upward from the stationary bottom endplate; at Monash, both endplates move 


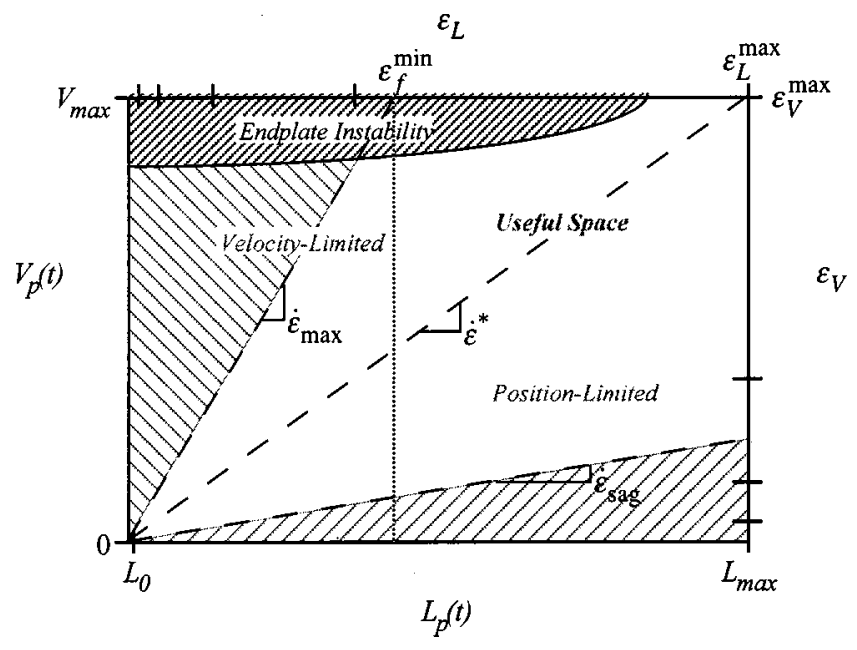

FIG. 6. Operating space of a generic filament stretching device. Motor and sensor limitations translate into parameter ranges in uniaxial extensional flow, shown here for an ideal experiment in which $L_{p}(t)=L_{0} \exp \left(\dot{\varepsilon}_{0} t\right)$. Fluid properties and geometry considerations can also lead to additional limitations due to gravitational sagging and endplate instabilities.

simultaneously in opposite directions away from the stationary midplane of the filament; and at Toronto, the bottom endplate moves downward from the stationary top endplate. Each configuration leads to a slightly different balance of forces on the fluid filament, the details of which will be discussed later in this section. In contrast to the other two instruments, in the Toronto device the filament diameter is measured at the filament midplane only up to a total plate separation of about $L_{p} \approx 48 \mathrm{~mm}$, at which time the diameter sensor stops moving. This method is expected to yield acceptable diameter measurements for at least strongly strain-hardening fluids because the filament diameter has been found to be uniform for dilute polymer solutions [Sridhar et al. (1991)].

In each device, the diameter sensor has a resolution of better than $50 \mu \mathrm{m}$, and the force transducer has a resolution of less than $0.1 \%$ of the maximum measurable force. Both diameter and force sensors are calibrated periodically at each laboratory using optical fibers of known diameter and calibration weights, respectively. Each force transducer has a different dynamical response, characterized by the rolloff frequency $f_{c}$ and the damping coefficient $\gamma_{D}$, given in Table III. The values of these parameters determine the characteristic time of the transducer to respond to transient loads. Fluctuations in the force data can be reduced using a low-pass filter. Finally, the initial aspect ratio of the fluid filament $\Lambda_{0} \equiv L_{0} /\left(D_{0} / 2\right)$ is approximately unity for each rheometer, leading to similar initial transient force responses as stretching begins [Spiegelberg et al. (1996)].

Geometric dimensions and motor capacity determine the ranges of experimental parameters accessible in a given device. Figure 6 depicts the generic operating space for a device, where endplate velocity is plotted as a function of endplate position. The maximum position $L_{\max }$ and the maximum velocity $V_{\max }$ achievable by the motors form the bounds of the operating space. An ideal uniaxial extensional flow, described by Eq. (21), is a straight line on this phase diagram, with a slope equal to the imposed strain rate $\dot{E}$. It is clear from the diagram that a given experiment will be limited by either the total travel available to the motor platens or by the maximum velocity the motors can provide. The optimum strain rate, $\dot{\varepsilon}^{*}=V_{\max } / L_{\max }$, is the rate which utilizes the full ranges of 
both position and velocity. For each experiment, the maximum achievable Hencky strain is determined by the limiting variable: if position-limited,

$$
\varepsilon_{\max }=\ln \left(\frac{L_{\max }}{L_{0}}\right),
$$

if velocity-limited,

$$
\varepsilon_{\max }=\ln \left(\frac{V_{\max }}{\dot{E} L_{0}}\right) .
$$

Conversely, the maximum stretch rate for a device is dictated by the desired final Hencky strain for a given experiment, and can be found by solving Eq. (23b) for $\dot{E}_{\max }$.

In addition to these considerations, it must be remembered that the motor responds in a finite time ( $\delta t \approx 50-100 \mathrm{~ms}$ ) to a motion command, further limiting the maximum controllable strain rate. The maximum stretching rate $\dot{\varepsilon}_{\max }$ that can be attained in a filament stretching device is ultimately determined by the limiting (i.e., slowest) response time $\delta t_{\max }$ of either the velocity-position feedback controller, the radial micrometer, or the force transducer. A (generous) estimate of this limiting strain rate can be found by considering an experiment of total duration $\delta t_{\max }$ which is also velocity limited so that at the end of the experiment we have

$$
V_{\text {max }}=V_{0} \exp \left(\dot{\varepsilon}_{\max } \delta t_{\max }\right) .
$$

Substituting for $V_{0}=\dot{\varepsilon}_{\max } L_{0}$ and rearranging leads to the following implicit expression for the maximum stretch rate of a given filament stretching device

$$
\left(\delta t_{\max }\right) \dot{\varepsilon}_{\max }+\ln \left(\dot{\varepsilon}_{\max }\right)=\ln \left(V_{\max } / L_{0}\right) \text {. }
$$

For a typical filament stretching device with $\delta t_{\max } \approx 0.050 \mathrm{~s}, V_{\max } \approx 2.0 \mathrm{~m} / \mathrm{s}$, and an initial sample size of $L_{0} \approx 2 \times 10^{-3} \mathrm{~m}$, the maximum stretch rate is thus $\dot{\varepsilon}_{\text {max }} \approx 57 \mathrm{~s}^{-1}$. Carefully controlled “type III"' experiments [Kolte et al. (1997)] at such stretch rates would be extremely difficult to realize in practice due to the very limited time available for communication between the linear stage, the radius measuring device, and the controller. These limits on the range of attainable Hencky strains and strain rates will affect whether a steady state extensional viscosity can be measured in a given experiment.

The procedure for performing a successful filament stretching experiment begins by determining the correct plate motion to impose. Although the endplate position profile $L_{p}(t)$ is nominally described by Eq. (21), the no-slip condition at the endplates introduces shearing there, which affects the midfilament diameter $D_{\text {mid }}(t)$. Several authors have examined this nonideal flow [Shipman et al. (1991); Spiegelberg et al. (1996); Yao and McKinley (1998)], and methods of performing and analyzing filament stretching experiments have been proposed by Kolte and coworkers [Kolte et al. (1997)]. It has been found that the optimal endplate separation is not a simple exponential function but rather a more complex motion such that the midfilament diameter decreases exponentially, according to

$$
D_{\text {mid }}=D_{0} \exp \left(-0.5 \dot{\varepsilon}_{0} t\right) .
$$

This so-called "type III" test [Kolte et al. (1997)] is straightforward to generate numerically but difficult to realize experimentally. In early work, the required endplate motion 
was obtained by trial-and-error. However, two equivalent methods of realizing this ideal diameter profile have recently been put forward [Anna et al. (1999); Orr and Sridhar (1999)], and these "master-curve" techniques are used in this study to achieve nearly ideal flow kinematics.

Once the correct endplate position history $L_{p}(t)$ has been determined [i.e., the one which results in a $D_{\text {mid }}(t)$ profile that decreases according to Eq. (26)], this motion profile is downloaded to the motion controller. A small volume of fluid is introduced into the gap between the endplates such that the fluid forms a right-circular cylinder of diameter $D_{0}$ and length $L_{0}$. While the fluid filament is being stretched, the force $F_{p}(t)$ and the midfilament diameter $D_{\text {mid }}(t)$ are measured simultaneously. Since the diameter decreases exponentially, the actual strain rate is determined by least-squares fitting of the logarithm of the diameter to a linear function of time.

In order to compute the transient extensional viscosity from the measurements, forces on a viscoelastic filament must be balanced. A detailed analysis of this force balance, incorporating surface tension, filament curvature, weight of the filament, inertia, and endplate acceleration, has been presented elsewhere [Szabo (1997)]. Because each laboratory has a different motion and force configuration, each applies a slightly different formula to compute tensile stresses. At M.I.T., where the force transducer is mounted to the stationary bottom endplate, the force balance yields

$$
\left\langle\tau_{z z}-\tau_{r r}\right\rangle=\frac{F_{p}}{\left(\pi D_{\mathrm{mid}}^{2} / 4\right)}+\frac{1}{2} \frac{\rho g V_{0}}{\left(\pi D_{\mathrm{mid}}^{2} / 4\right)}-\frac{\sigma}{\left(D_{\mathrm{mid}} / 2\right)}+\frac{1}{2} \frac{\rho V_{0} \ddot{L}_{p}}{\left(\pi D_{\mathrm{mid}}^{2} / 4\right)},
$$

where $\left\langle\tau_{z z}-\tau_{r r}\right\rangle$ is the principal tensile stress difference in the fluid filament, $V_{0}=\pi L_{0}\left(D_{0} / 2\right)^{2}$ is the volume of the fluid sample, $\sigma$ is the surface tension of the fluid, and $\rho$ is the density of the fluid. At Toronto, where the force transducer is mounted to the stationary top endplate, the sign of the second term in Eq. (27) is negative. And at Monash, where the force transducer moves with the top plate and both plates move apart, the Toronto equation is used without the final $\ddot{L}_{p}$ term arising from fluid inertia. In practice, this inertial term is found to contribute less than $1 \%$ to the stress difference, so this term is not actually computed. From Szabo's detailed force balance analysis, we neglect terms involving axial filament curvature $R_{s}^{\prime \prime}$ and other inertial terms because filaments of dilute polymer solutions become increasingly uniform along their length during stretching [Szabo (1997)]. The force balance given in Eq. (27) assumes that the force transducer has been tared prior to loading the fluid sample.

Finally, the dimensionless extensional viscosity, or Trouton ratio $(\mathrm{Tr})$ is computed, utilizing time-temperature superposition when necessary, by the following expression:

$$
\operatorname{Tr}=\frac{\bar{\eta}^{+}(T)}{a_{T} \eta_{0}}=\frac{\left\langle\tau_{z z}-\tau_{r r}\right\rangle}{\dot{\varepsilon}_{0} a_{T} \eta_{0}}
$$

where $\dot{\varepsilon}_{0}$ is obtained from the fit to the raw diameter data, and $a_{T}$ is given by Eq. (8). The Hencky strain at the midplane of the filament is computed using the measured mid-filament diameter

$$
\varepsilon_{D} \equiv-2 \ln \left(D_{\text {mid }}(t) / D_{0}\right)=\dot{\varepsilon}_{0} t .
$$

Investigators at each laboratory independently verified that their measurements of the Trouton ratio are reproducible for identical conditions. Errors are a major concern in experimental work, of course, and thus we examined the contribution of various sources of error by performing standard error propagation analyses using Eqs. (27) and (28). 
Since the first term in the force balance equation is the dominant one during stretching, we restrict our analysis to this term only. The error in the Trouton ratio as a function of the Hencky strain is given by

$$
\frac{\delta \operatorname{Tr}}{\operatorname{Tr}}=\left\{\left(\frac{\delta F}{F}\right)^{2}+\left(\frac{2 \delta D}{D}\right)^{2}+\left(\frac{\delta a_{T}}{a_{T}}\right)^{2}+\left(\frac{\delta \dot{\varepsilon}_{0}}{\dot{\varepsilon}_{0}}\right)^{2}\right\}^{1 / 2},
$$

where $\delta F$ is the magnitude of fluctuations in a baseline force signal, $\delta D$ is the deviation from the ideal diameter profile given in Eq. (26), $\delta a_{T}$ is the temperature measurement error, and $\delta \dot{\varepsilon}_{0}$ is the standard deviation resulting from the least-squares fit to the strain rate as described earlier. Each term in Eq. (30) can contribute significantly at different times in the stretching. Although $\delta a_{T} / a_{T}$ and $\delta \dot{\varepsilon}_{0} / \dot{\varepsilon}_{0}$ are generally constant during a given experiment, $\delta F / F$ and $\delta D / D$ both vary with time. Calculations using Eq. (30) will be given for a specific experiment in Sec. III C. Finally, we note that experiments were performed on the M.I.T. device using both $D_{0}=3.0 \mathrm{~mm}$ and $D_{0}=7.0 \mathrm{~mm}$ endplates, with identical initial aspect ratios $\Lambda_{0}=1.0$ for each experiment. The resulting values of $\mathrm{Tr}$ agreed well until late times, when the filament diameter fell below the resolution of the diameter sensor and produced unreliable and noisy data.

The operating conditions of a particular experiment can be characterized by several dimensionless groups. The most important of these is the Deborah number De, which is the dimensionless extensional rate, defined by

$$
\text { De }=\lambda_{Z} \dot{\varepsilon}_{0} .
$$

Another dimensionless group, the Bond number Bo, describes the competition between gravity, which causes the filament to sag and drain, and surface tension, which acts to maintain the cylindrical shape of the filament. The Bond number is given by

$$
\text { Bo }=\frac{\rho g D_{0}^{2}}{4 \sigma} \text {. }
$$

This number is useful because it is a measure of the axial asymmetry of the initial static fluid column about its midplane. Large Bond numbers, Bo $>1$, indicate that surface tension is not able to maintain a perfectly cylindrical fluid filament; in practice, a visible bulge appears in the fluid below the midplane, which increases in size as Bo increases [Szabo (1997); Domann and McKinley (1998)]. Another dimensionless group related to surface tension is the capillary number $\mathrm{Ca}$, which describes the competition between viscous forces and surface tension

$$
\mathrm{Ca}=\frac{\eta_{0} \dot{\varepsilon}_{0} D_{0}}{2 \sigma} .
$$

For Newtonian fluids at least, the capillary number is a measure of the stability of the slender fluid filament as it is stretched. Viscous forces in the filament tend to stabilize the cylindrical shape, while surface tension acts to destabilize the shape by causing the diameter to rapidly decrease until the filament breaks apart [Ide and White (1976); Hassager et al. (1998b)].

The ratio of the Bond number to the capillary number

$$
\frac{\mathrm{Bo}}{\mathrm{Ca}}=\frac{\rho g D_{0}}{2 \eta_{0} \dot{\varepsilon}_{0}}
$$


expresses the competition between gravitational and viscous forces during stretching. A large value of $\mathrm{Bo} / \mathrm{Ca}$ indicates that a significant amount of fluid drains below the midplane of the filament during stretching. In strongly strain-hardening fluids, draining is not an issue because stresses in the filament are of the order $\bar{\eta}^{+} \dot{\varepsilon}_{0}$ rather than $\eta_{0} \dot{\varepsilon}_{0}$. At lower stretch rates, however, sagging can be important. Nevertheless, in computing the transient extensional viscosity from Eqs. (27) and (28), it is assumed that exactly half the volume of the fluid filament lies below the midplane [Szabo (1997)]. If $\mathrm{Bo} / \mathrm{Ca} \geqslant 1$, significantly more than $50 \%$ of the fluid volume will drain below the midplane, leading to an additional correction term in Eq. (27) of the form

$$
\frac{\rho g \delta V}{\left(\pi D_{\text {mid }}^{2} / 4\right)}
$$

where $\delta V$ is the extra volume below the midplane. The evolution of this incremental volume with time is a complicated function of surface curvature and cannot be computed analytically even for a Newtonian filament. Although the correction term in Eq. (35) is not easy to calculate, it can become large, and extensional viscosities computed without accounting for it exhibit anomalously low values at moderate Hencky strains, even becoming negative for large enough $\mathrm{Bo} / \mathrm{Ca}$ values.

The experimental conditions for which gravitational sagging will become important can be estimated using dimensionless quantities. Since gravitational forces begin to dominate when the ratio Bo/Ca approaches unity, Eqs. (34) and (8) can be used to compute a critical strain rate $\dot{\varepsilon}_{\text {sag }}$ below which sagging becomes important

$$
\dot{\varepsilon}_{\mathrm{sag}}=\frac{\rho g D_{0}}{2 \eta_{0} a_{T}} .
$$

This condition leads to a critical Deborah number De $_{\text {sag }}$, which depends on material properties and test geometry and which is only a weak function of temperature [cf. Eq. (8)]:

$$
\mathrm{De}_{\text {sag }}=\lambda_{Z} \frac{\rho g D_{0}}{2 \eta_{0}} \frac{T_{0}}{T} .
$$

Values of the dimensionless groups Bo and $\mathrm{De}_{\text {sag }}$ are given in Table III for each rheometer and for the three SM test fluids at the reference temperature $T_{0}$. The Deborah number for a given experiment can be compared to $\mathrm{De}_{\mathrm{sag}}$ to determine if sagging effects are important; Deborah numbers greater than $\mathrm{De}_{\mathrm{sag}}$ are expected to be less affected by sagging. For the SM fluids, it was shown in Sec. II B that the longest relaxation time $\lambda_{Z}$ increases much more rapidly with molecular weight than does the total shear viscosity $\eta_{0}$; hence Eq. (37) shows that the potential for sagging increases not only with the plate size but also with molecular weight.

Gravitational sagging is a particularly important constraint in filament stretching devices if one wishes to explore experimentally the coil-stretch transition in dilute polymer solutions at Deborah numbers of $\mathrm{De} \approx 0.5$. This may be demonstrated by considering the critical Deborah number for sagging in more detail. For a dilute polymer solution described by the Zimm theory, the longest relaxation time and polymer contribution to the viscosity may be expressed as $\lambda_{Z}=2.369[\eta] \eta_{s} M_{w} /\left(N_{A} k_{B} T\right)$ and $\eta_{p} \equiv\left(\eta_{0}-\eta_{s}\right)$ $=[\eta] \eta_{s} c$, respectively. The critical Deborah number for sagging can thus be written in the compact form 


$$
\mathrm{De}_{\mathrm{sag}} \equiv \frac{\lambda_{Z} \rho g D_{0}}{2 \eta_{0}}=\frac{2.369[\eta] M_{w} \rho g\left(D_{0} / 2\right)}{\left(N_{A} k_{B} T_{0}\right)(1+[\eta] c)} .
$$

The intrinsic viscosity can also be represented in the form of the Mark-HouwinkSakaruda expression $[\eta]=K^{\prime} M_{w}^{a^{\prime}}$ (in which $K^{\prime}$ and $a^{\prime}$ are tabulated constants for a particular polymer-solvent pair). For the solution to be considered dilute we require $c[\eta] \leqslant 1$, which places an upper bound on the denominator. If we require $\mathrm{De}_{\mathrm{sag}}$ $\leqslant 0.5$ then Eq. (38) can be reduced to

$$
\frac{1.18\left(K^{\prime} M_{w}^{1+a^{\prime}}\right) \rho g\left(D_{0} / 2\right)}{R T_{0}} \leqslant 0.5,
$$

where $R=N_{A} k_{B}$ is the ideal gas constant.

For most polymer solutions in good solvents, the constant $K^{\prime}$ is in the range (10 $\left.\leqslant K^{\prime} \leqslant 50\right) \times 10^{-3} \mathrm{ml} / \mathrm{g}$ [see e.g., Brandrup et al. (1999)]. Taking typical values of $\rho g \approx 10^{4} \mathrm{~J} / \mathrm{m}^{4}, R T_{0} \approx 2400 \mathrm{~J} / \mathrm{mol}$ and $D_{0} / 2 \approx 10^{-3} \mathrm{~m}$ we thus need to perform experiments with fluids having relative molecular masses in the range

$$
(0.5-2.5) \times 10^{-10}\left(M_{w}\right)^{1+a^{\prime}} \leqslant 0.5
$$

The Zimm theory gives $\left(1+a^{\prime}\right) \equiv 3 \nu$, where $\nu$ is the scaling exponent for the coil size determined from light scattering. For example, from Table I we find $\nu \approx 0.52$ and $K \approx 35 \times 10^{-3} \mathrm{ml} / \mathrm{g}$ for the PS/PS fluids employed in this study. We would therefore need to use polymers with molecular weights less than $M_{w} \leqslant 1.15 \times 10^{6} \mathrm{~g} / \mathrm{mol}$ in order to explore the coil-stretch transition in the absence of appreciable gravitational sagging.

It is interesting to note that although this result does depend on the solvent quality parameter $\nu$, it is independent of the viscosity of the solvent (since both the longest relaxation time and the polymer contribution vary linearly on $\eta_{s}$ ). Changing the solvent viscosity does, however, change the absolute magnitude of the tensile force exerted on the endplates during the stretching. This places another independent constraint on the experimental range attainable by filament stretching devices.

\section{B. Realization of kinematics}

This component of the study was undertaken with the hope that, in spite of slight variations in instrument configuration, different laboratories can achieve the same results for identical fluids. Before we compare our results from the different instruments, we first examine how closely the ideal stretching history is achieved.

As mentioned in the previous section, the first step in a successful filament stretching experiment is to determine the correct kinematics, i.e., the endplate separation $L_{p}(t)$ and velocity $\dot{L}_{p}(t)$ that result in the midfilament diameter decreasing exponentially [Eq. (26)]. We use the techniques developed at M.I.T. and Monash to determine these kinematics [Anna et al. (1999); Orr and Sridhar (1999)]. Figure 7 shows a so-called "master curve", in which one measure of the total Hencky strain is plotted against the other, the two Hencky strains being given by Eqs. (22) and (29). The nonlinearity of this plot reflects the influence of the no-slip boundary condition at the endplates and the extent of the strain hardening in the fluid rheology. A filament which is deforming ideally is cylindrical along its entire length for the duration of the experiment, and then the Hencky strain based on endplate separation would exactly equal the Hencky strain based on midfilament diameter. In reality, they are not equal. However, for sufficiently high strain rates, the curve should be independent of the kinematics for a particular test fluid, as suggested by 


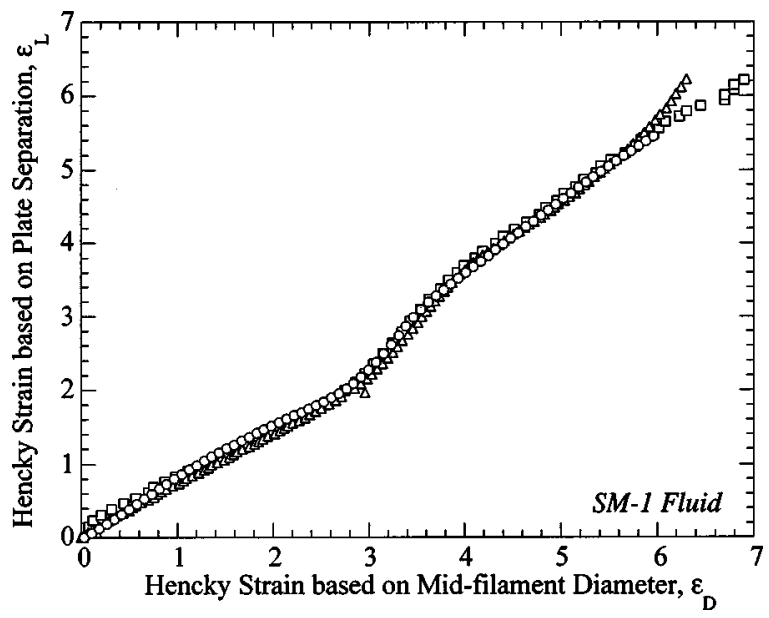

FIG. 7. Comparison of the "master curves" for SM-1 from the three filament stretching devices at similar Deborah numbers. Good agreement is found between the Hencky strain profiles measured by M.I.T. ( $\bigcirc$, $\mathrm{De}=17.0)$, Monash $(\square, \mathrm{De}=14.0)$, and Toronto $(\triangle, \mathrm{De}=12.0)$.

extensive experimentation and constitutive arguments [Anna et al. (1999); Orr and Sridhar (1999)]. The plot can thus be used to invert a nonideal experiment, in which the endplate position is described by Eq. (21), into a nearly ideal experiment, in which the midfilament diameter is described by Eq. (26). Details of the inversion process are described elsewhere [Anna et al. (1999); Orr and Sridhar (1999)]. The curves shown in Fig. 7 are data from the three laboratories for SM-1 at similar Deborah numbers. The three curves are very close, indicating that the imposed kinematics are virtually the same at the three laboratories. Each laboratory found that the curve actually is weakly dependent on the Deborah number, and so this "master curve" must be recreated for each imposed strain rate [Anna et al. (1999)].

We further investigate the kinematics by comparing the endplate positions and velocity profiles achieved by the individual motors. Since the linear motor(s) in each rheometer controls either position or velocity, but not both, plotting an operating diagram similar to that in Fig. 6 would allow a proper comparison. However, since the initial axial separation $L_{0}$ is different at each laboratory, and since the imposed strain rates $\dot{\varepsilon}_{0}$ are also slightly different, the endplate velocity is normalized by the characteristic velocity $\dot{\varepsilon}_{0} L_{0}$ and the endplate position by the initial separation $L_{0}$. (This type of operating diagram essentially plots a third characteristic Hencky strain, based on the endplate velocity, as a function of the Hencky strain based on position.) The results are shown in Fig. 8, for the same data presented in Fig. 7. Again, we observe that the kinematics realized by the different devices agree reasonably well. We have found that quantitative agreement between imposed velocity and position profiles is crucial in obtaining good agreement between transient Trouton ratio profiles. Fluctuations in the low velocity data shown for the Monash and Toronto devices result from low resolution in the velocities reported by the motion controller, although the actual velocities are observed to be smooth. The velocity profile shown for the M.I.T. device is smoother because it is computed during postprocessing by fitting a spline curve to the reported endplate position data.

Finally, we can examine how successfully the modified kinematics achieve the desired midfilament diameter profiles. Figure 9(a) shows the actual midfilament diameter profiles from the three laboratories along with the ideal diameter, the solid line, given by Eq. (26). 


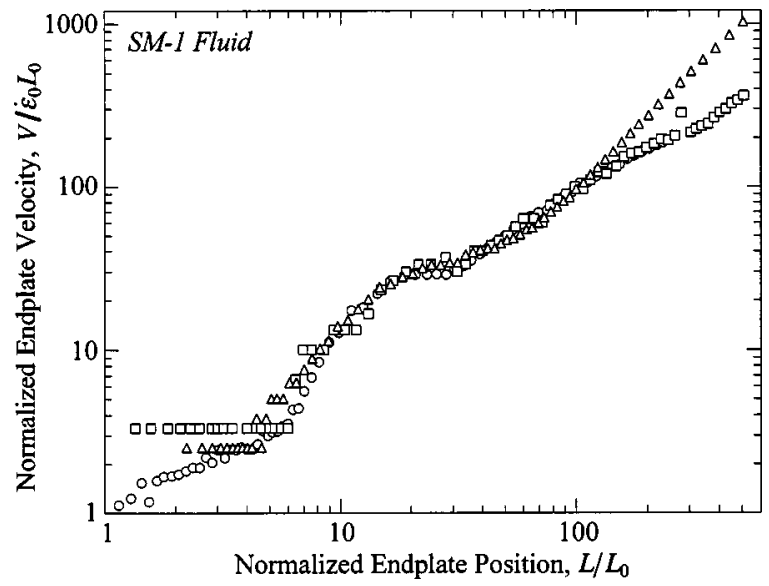

FIG. 8. Comparison of endplate kinematics with the SM-1 fluid [M.I.T. ( $\bigcirc$, De $=17.0)$, Monash $(\square$, De $=14.0)$, and Toronto $(\triangle, \mathrm{De}=12.0)]$.

These curves appear to show excellent agreement, as they are nearly indistinguishable from the ideal curve on this scale. A more illuminating plot is shown in Fig. 9(b), which shows diameter error. This error measure is defined as the deviation of the actual midfilament diameter from the ideal value and normalized by the ideal value so that

$$
\frac{\delta D}{D}=\frac{D_{\text {ideal }}-D_{\text {mid }}}{D_{\text {ideal }}}=1-\frac{D_{\text {mid }}}{D_{0} e^{-0.5 \varepsilon_{0}}} .
$$

Figure 9(b) shows that all three laboratories are able to maintain an error of less than 10\% for the duration of a run. Although the error plot has a distinct shape for each laboratory, showing that the errors are not random, the errors are small and not expected to significantly affect the resulting transient extensional viscosities. The excellent agreement between imposed endplate kinematics and resulting midfilament diameter profiles from the three laboratories leads us to expect similarly good agreement between the transient extensional viscosities computed with the three devices. These viscosities are presented in Sec. IV.

\section{EXTENSIONAL RHEOLOGY OF SM FLUIDS}

Having imposed proper flow kinematics, we now compare transient extensional viscosities from the three laboratories. Figure 10 shows the transient Trouton ratios for SM-1 fluid after appropriate versions of Eqs. (27) and (28) are applied. As we had hoped, the Trouton ratio profiles agree very well, consistent with the good agreement between endplate kinematics and midfilament diameter profiles in Figs. 7, 8, and 9. The shape of the Trouton ratio profile is also consistent with previously reported measurements of the transient extensional rheology of dilute polymer solutions [Sridhar et al. (1991); Kröger et al. (1992); Tirtaatmadja and Sridhar (1993); Berg et al. (1994); Solomon and Muller (1996b); Spiegelberg et al. (1996); Spiegelberg and McKinley (1996); van Nieuwkoop and Muller von Czernicki (1996); Jain et al. (1997); Remmelgas et al. (1998); Anna et al. (1999); Orr and Sridhar (1999); Verhoef et al. (1999)]. Figure 10 shows that the fluid response is primarily viscous initially, for the Trouton ratio has a constant value of approximately 3, consistent with the value expected for a purely Newtonian fluid [Trouton (1906)]. For Boger fluids, the initial Trouton ratio should be slightly lower than 3, 

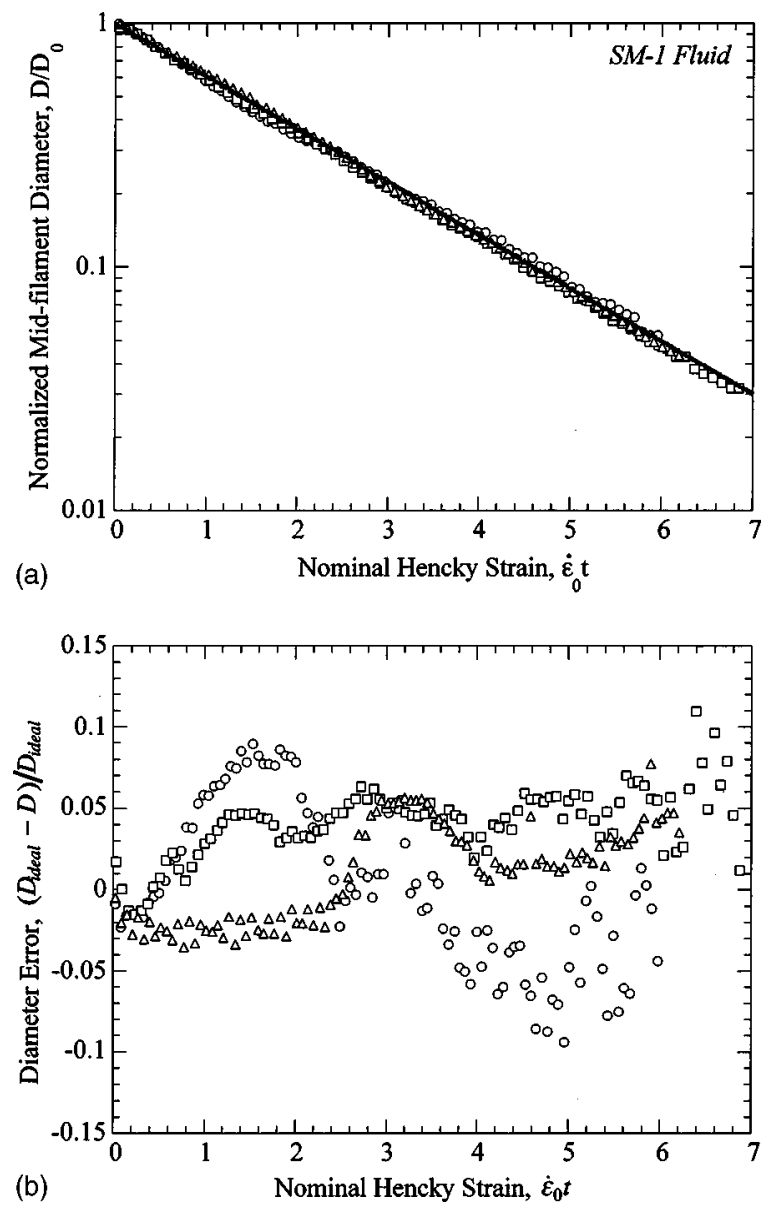

FIG. 9. Comparison of (a) midfilament diameter and (b) diameter error for SM-1. In (a) the midfilament diameter measurements from all three laboratories [M.I.T. $(\bigcirc$, De $=17.0)$, Monash $(\square$, De $=14.0$ ), and Toronto $(\triangle, \mathrm{De}=12.0)]$ are nearly indistinguishable from the ideal profile (solid line). The diameter errors in (b) show that all three filament stretching devices produce diameters within $10 \%$ of the ideal value during stretching.

with a value of $3 \eta_{s} / \eta_{0}$, since the total viscosity $\eta_{0}$ is larger than the solvent viscosity $\eta_{s}$. At moderate strains of about $\varepsilon \sim 2$, the polymer chains are stretched significantly, strain hardening begins to occur, and the Trouton ratio begins to rise. Finally, at Hencky strains of about $\varepsilon \sim 6$, the polymer chains are nearly fully stretched, and the Trouton ratio approaches a steady state value $\operatorname{Tr}_{\infty}$. For SM-1, gravitational effects are found to be unimportant because the experimental Deborah numbers are considerably larger than the critical Deborah number $\mathrm{De}_{\text {sag }}$.

Error bars at selected strains have been computed from Eq. (30) and are also included in Fig. 10. At low Hencky strains, such as those near (a) in the plot, viscous forces are large and diameter values are well above sensor resolution; hence the error bars are less than 5\% of the total Trouton ratio in this region. At moderate Hencky strains (b), the viscous force has decayed significantly and strain hardening has not yet begun; hence the measured force is a minimum and possibly close to the resolution of the transducer. In this region, errors can be as high as 50\% of the transient Trouton ratio, depending on the 


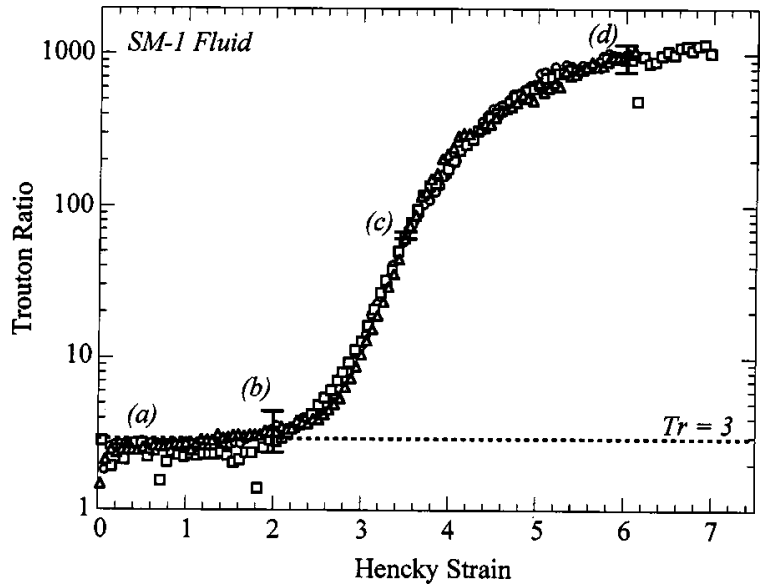

FIG. 10. Comparison of transient Trouton ratio profiles for SM-1 [M.I.T. $\left(\bigcirc, \mathrm{De}=17.0, T=21.3{ }^{\circ} \mathrm{C}\right)$, Monash $\left(\square, \mathrm{De}=14.0, T=19.5^{\circ} \mathrm{C}\right)$, and Toronto $\left.\left(\triangle, \mathrm{De}=12.0, T=21.6^{\circ} \mathrm{C}\right)\right]$. The bars indicate the estimated error at low, moderate, and high Hencky strains.

resolution of the force transducer. Once the force begins to increase due to strain hardening at strain (c), the error again becomes small and, for Hencky strains of about $\varepsilon \sim 3-4$, the error bars are less than 5\%. Finally, near the end of the experiment at strain (d), the force is decreasing and the diameter is approaching the resolution of the sensor. In this region, error bars can reach $15 \%-20 \%$ of the transient $\operatorname{Tr}$ value.

In addition to random fluctuations in the measured data, systematic errors can arise. We have found that it is essential to calibrate the force and diameter sensors frequently and carefully, and to ensure that the dynamical response of each sensor is fast enough that no noticeable delay is introduced in the signal. The temperature needs to be monitored frequently and recorded immediately prior to stretching because the fluid viscosities, and therefore the relaxation times, are extremely sensitive to temperature, as shown by Eq. (8). An error in the temperature shifts the entire Tr curve by a constant factor, which increases rapidly as the magnitude of the temperature error increases. Other important considerations for reproducible data include: ensuring that the endplates are clean, axially aligned, and parallel; loading the sample such that the initial liquid bridge is free of bubbles; and obtaining accurate measurements of density, surface tension, and viscometric properties.

Figures 11 and 12 compare filament stretching data for similar Deborah numbers for the SM-2 and SM-3 fluids, respectively. For SM-2, the data from Monash and Toronto agree quite well. However, the effect of gravitational sagging is significant in the M.I.T. data for the reason described in the text after Eq. (37), and this curve does not begin to approach the other two curves until Hencky strains of about $\varepsilon \sim 4$. The ratios of the experimental Deborah number to the critical Deborah number for each laboratory are De/De $\mathrm{sag}_{\mathrm{s}}=0.64,1.5$, and 3.1 for M.I.T., Monash, and Toronto, respectively, and these values help to explain the observed trends. The ratio is significantly smaller than unity for M.I.T., indicating greater potential for sagging. The ratio is $O(1)$ for the other two laboratories, suggesting that gravity should not play an observable role. For SM-3, sagging is again significant for the M.I.T. data, although the ratio of the Deborah numbers is about $\mathrm{De} / \mathrm{De}_{\mathrm{sag}}=1.2$ in this case. Agreement between the Monash and Toronto data is not quite as good in this case, and the discrepancy can be explained by sagging, since the Deborah number ratios are $\mathrm{De} / \mathrm{De}_{\mathrm{sag}}=0.76$ (Monash) and 2.6 (Toronto). Based 


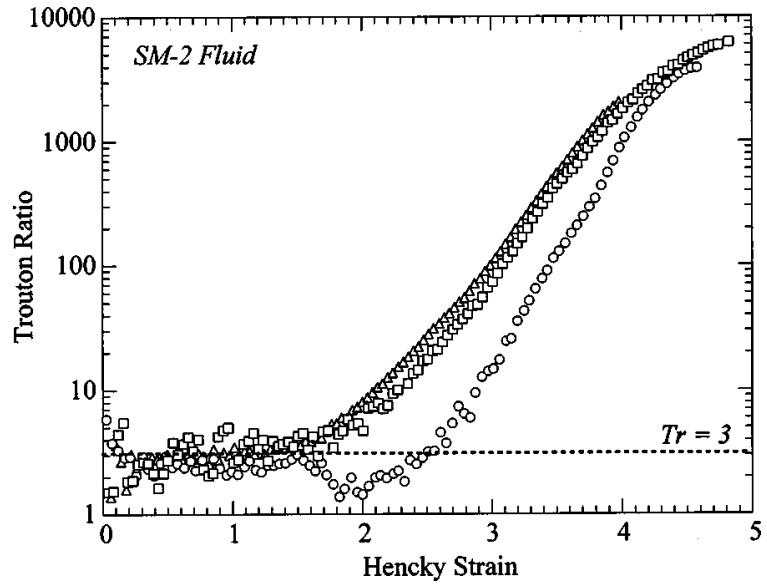

FIG. 11. Comparison of transient Trouton ratio profiles for SM-2 [M.I.T. $\left(\mathrm{O}, \mathrm{De}=15.2, T=25.3{ }^{\circ} \mathrm{C}\right)$, Monash $\left(\square, \mathrm{De}=15.6, T=21.0^{\circ} \mathrm{C}\right)$, and Toronto $\left.\left(\triangle, \mathrm{De}=31.8, T=27.5^{\circ} \mathrm{C}\right)\right]$. The effect of gravitational sagging is evident in the experiments performed at M.I.T. in which larger endplates were used.

on these ratios, one might expect the sagging effect in both the M.I.T. and Monash data to have nearly the same magnitude. We believe other factors influence the transient evolution of these two curves, namely the Bond number Bo and the initial aspect ratio $\Lambda_{0}$, which are not captured in the simple dimensionless criterion of Eq. (37). For the M.I.T. experiments, Bo and $\Lambda_{0}$ are both significantly larger than those for the Monash experiments. These combined factors result in more asymmetry in the initial fluid column, which propagates into the force balance during stretching.

No laboratory was able to measure Trouton ratios beyond Hencky strains of about $\varepsilon \sim 4-5$, for either SM-2 or SM-3, because of an elastic instability near the endplates. This instability has been observed and characterized previously for polystyrene- and polyisobutylene-based Boger fluids [Spiegelberg and McKinley (1996); Rasmussen and Hassager (1999)]. Its onset is preceded by a rapid drainage of the fluid adjacent to the endplates, leading to a large negative pressure gradient across the free surface of the

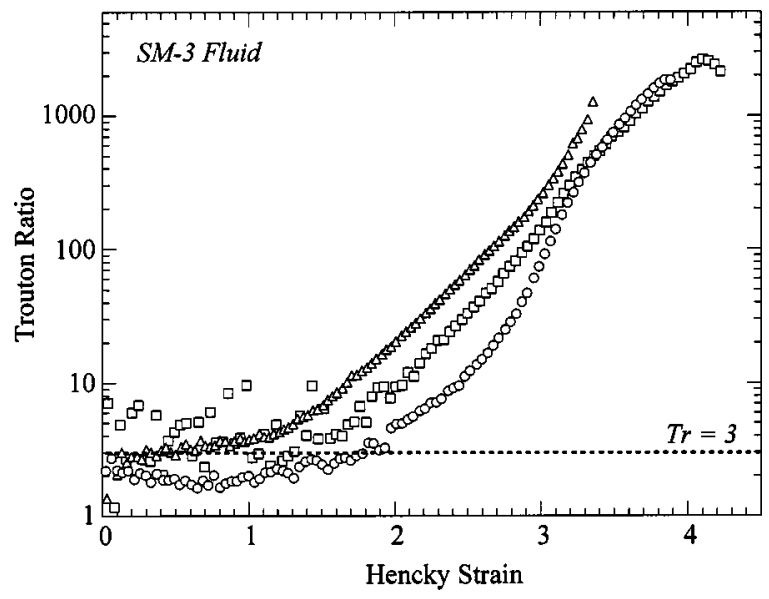

FIG. 12. Comparison of transient Trouton ratio profiles for SM-3 [M.I.T. $\left(\bigcirc\right.$, De $\left.=114, T=23.1^{\circ} \mathrm{C}\right)$, Monash $\left(\square, \mathrm{De}=31.8, T=25.0^{\circ} \mathrm{C}\right)$, and Toronto $\left.\left(\triangle, \mathrm{De}=110, T=28.8^{\circ} \mathrm{C}\right)\right]$. 


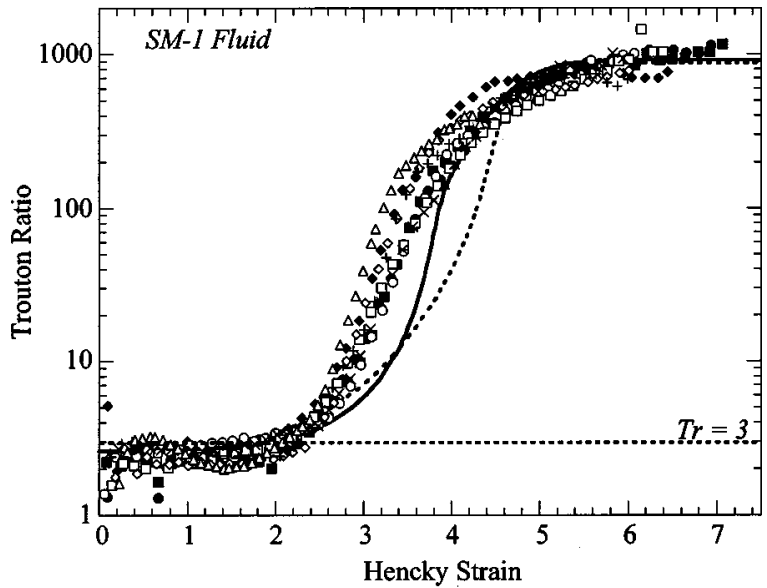

FIG. 13. Transient extensional rheology of SM-1 fluid over a range of Deborah numbers. Data from all three filament stretching devices are represented [M.I.T. $\left(\times, \mathrm{De}=17.0, T=21.3{ }^{\circ} \mathrm{C} ;+, \mathrm{De}=27.2, T=22 .{ }^{\circ} \mathrm{C}\right.$; $\triangle$, De $\left.=71.0, T=22.1{ }^{\circ} \mathrm{C}\right)$, Monash $\left(\bullet, \mathrm{De}=14.0, T=19.5^{\circ} \mathrm{C} ; \boldsymbol{\square}, \mathrm{De}=28.2, T=19.5^{\circ} \mathrm{C} ; \bullet, \mathrm{De}\right.$ $\left.=47.6, T=21.0^{\circ} \mathrm{C}\right)$, and Toronto $\left(\bigcirc, \mathrm{De}=12.0, T=21.6^{\circ} \mathrm{C} ; \square, \mathrm{De}=19.5, T=22.5^{\circ} \mathrm{C} ; \diamond, \mathrm{De}=46.7\right.$, $\left.\left.T=23.5^{\circ} \mathrm{C}\right)\right]$. Predictions from the FENE-PM model are also shown for both a high Deborah number and a low Deborah number $(\mathrm{De}=10$, dashed line; $\mathrm{De}=50$, solid line).

liquid "foot" and a destabilization of the azimuthal uniformity of the filament crosssection at large Hencky strains. These perturbations at the foot grow until the filament splits into "fingers," which elongate and move radially outward as the endplates continue to separate. For highly elastic fluids, the filament can completely detach from the endplates, and this occurs at all Deborah numbers for both fluids. Similar fibrillation is observed in adhesive materials [Ferguson et al. (1997); Crosby and Shull (1999)]. The detachment occurs at strains less than $\varepsilon \sim 5$, which prevents an approach to steady state conditions for these fluids, and thus prevents determination of the dependence of steady state Trouton ratios on polymer molecular weight. This elastic instability also limits our ability to compare transient curves with predictions from constitutive models.

In Figs. 13, 14, and 15 we show data for all three fluids over ranges of Deborah numbers. The transient Trouton ratios for SM-1, shown in Fig. 13, agree well when the Deborah numbers are similar in magnitude, as observed previously in Fig. 10. In addition, the Trouton ratio is seen to depend weakly on De, with the slope of the curve at moderate strains increasing slightly as De increases. Steady-state plateaus were apparent for several values of De, and the behavior of these plateaus will be discussed later. The Deborah numbers in Fig. 13 are larger than the critical Deborah number $\mathrm{De}_{\mathrm{sag}}$, and thus sagging has no effect.

Predictions from the FENE-PM bead-spring model (discussed in Sec. II B) are also included in Fig. 13, for two values of De which bound the experimental data. As shown in the figure, the predictions of the initial and steady state Trouton ratios agree well with the experimental values. However, the predicted evolution to steady state does not agree well. The onset of strain hardening occurs too late in the FENE-PM model, and the Peterlin approximation (FENE-P model) leads to an anomalously sharp approach to steady state. The dependence on De for this model does follow the experimentally observed trend, with the slope of the curve at moderate Hencky strains increasing with Deborah number. 


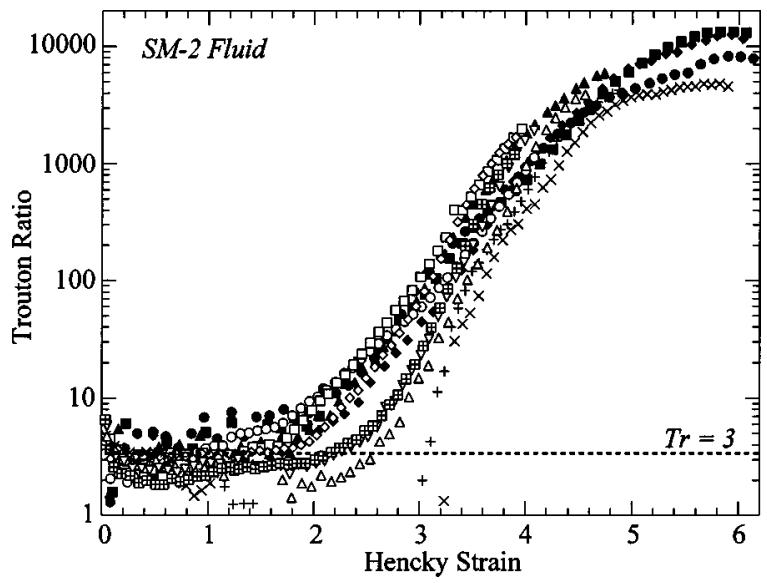

FIG. 14. Transient extensional rheology of SM-2 fluid over a range of Deborah numbers. Data from all three filament stretching devices are represented [M.I.T. $\left(\times, \mathrm{De}=3.3, T=24.7^{\circ} \mathrm{C} ;+, \mathrm{De}=8.0, T=25.1^{\circ} \mathrm{C} ; \triangle\right.$, $\left.\mathrm{De}=15.2, T=25.3^{\circ} \mathrm{C} ; \nabla, \mathrm{De}=26.4, T=25.4^{\circ} \mathrm{C} ; \boxplus, \mathrm{De}=58.3, T=22.7^{\circ} \mathrm{C}\right)$, Monash $(\bullet, \mathrm{De}=5.4$, $T=21.0^{\circ} \mathrm{C} ; \boldsymbol{\square}, \mathrm{De}=8.2, T=21.0^{\circ} \mathrm{C} ; \bullet, \mathrm{De}=10.9, T=21.0^{\circ} \mathrm{C} ; \boldsymbol{\Delta}, \mathrm{De}=15.6, T=21.0^{\circ} \mathrm{C}$ ), and Toronto $\left.\left(\mathrm{O}, \mathrm{De}=20.9, T=29.3^{\circ} \mathrm{C} ; \square, \mathrm{De}=31.8, T=27.5^{\circ} \mathrm{C} ; \diamond, \mathrm{De}=58.5, T=26.8^{\circ} \mathrm{C}\right)\right]$.

The FENE-PM model underpredicts the stress at intermediate strains $3 \leqslant \varepsilon \leqslant 4$. Brownian dynamics calculations [Doyle et al. (1998); Larson et al. (1999)] of bead-rod and bead-spring chains show that this stress discrepancy can arise from nonequilibrium internal configurations of the chain, which become trapped and thus develop "kinks" during strong deformations. The stress associated with such configurations leads to the stress-orientation hysteresis which has been observed in polymer solutions but which cannot be captured by closed-form models such as the FENE-PM model [Doyle et al. (1998)].

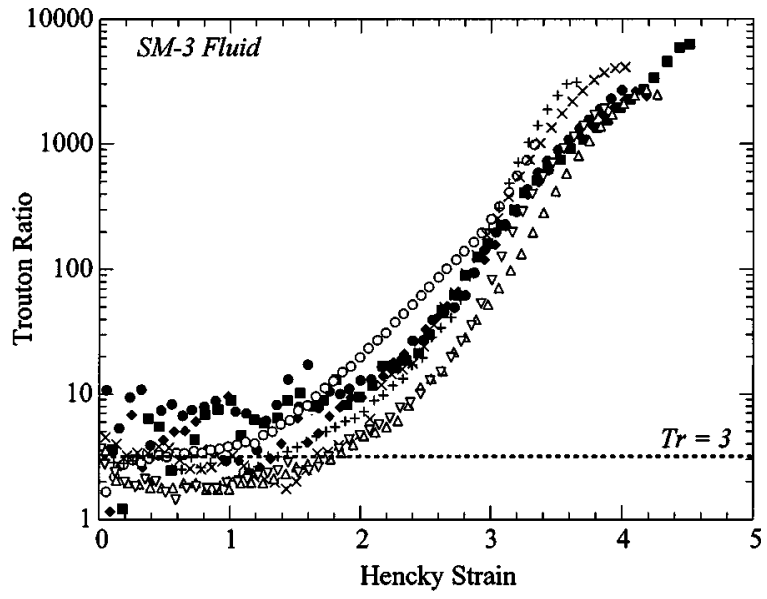

FIG. 15. Transient extensional rheology of SM-3 fluid over a range of Deborah numbers. Data from all three filament stretching devices are represented [M.I.T. $\left(\times, \mathrm{De}=21.2, T=23.6{ }^{\circ} \mathrm{C} ;+, \mathrm{De}=34.7, T=24.1{ }^{\circ} \mathrm{C}\right.$; $\triangle$, De $\left.=83.9, T=23.9^{\circ} \mathrm{C} ; \nabla, \mathrm{De}=114, T=23.1{ }^{\circ} \mathrm{C}\right)$, Monash $\left(\bullet, \mathrm{De}=10.8, T=25.0^{\circ} \mathrm{C} ; \mathbf{\square}, \mathrm{De}\right.$ $\left.=15.3, T=25.0^{\circ} \mathrm{C} ; \bullet, \mathrm{De}=31.8, T=25.0^{\circ} \mathrm{C}\right)$, and Toronto $\left.\left(\bigcirc, \mathrm{De}=110, T=28.8^{\circ} \mathrm{C}\right)\right]$. 


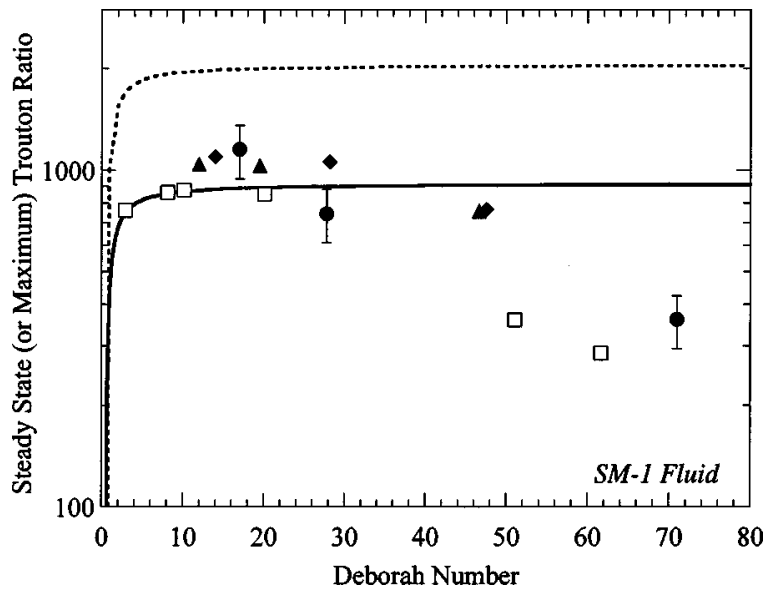

FIG. 16. Steady-state Trouton ratios for SM-1 fluid as a function of Deborah number. Values extracted from filament stretching experiments are shown for each device (M.I.T., $\bullet$; Monash, $\bullet$; and Toronto, $\mathbf{\Delta}$ ), and these experimental values agree to within 1 standard deviation. Theoretical predictions from the FENE-P model and the FENE-PM model are shown by the dashed and solid lines, respectively.

The transient Trouton ratios for SM-2 and SM-3 in Figs. 14 and 15 exhibit similar trends to those found for SM-1. However, as observed in Figs. 11 and 12, sagging plays a significant role in the data reported from M.I.T., and may also play a minor role in the data reported from Monash. As the Deborah number increases, the sagging effect in the M.I.T. data decreases, and the transient Trouton ratio curves begin to look similar to the curves reported from Monash and Toronto. As observed for SM-1, the curves for both SM-2 and SM-3 become steeper at moderate Hencky strains as De increases. The Hencky strain achieved in each experiment for SM-2 and SM-3 was limited by the fingering instability and filament detachment described earlier in this section. Only one experiment (performed at M.I.T.) reached a steady state value for SM-2, and this one corresponded to the lowest $\mathrm{De}$, with $\mathrm{De} / \mathrm{De}_{\mathrm{sag}}=0.14$. Without additional steady state values, it is unclear whether this single value is valid or is artificially low because of gravity.

Although steady state $\mathrm{Tr}$ values could not be observed for the two most elastic SM fluids due to elastic instability, values of $\operatorname{Tr}_{\infty}$ could be extracted over a range of De for SM-1. Figure 16 shows the extracted values. Error bars have been included for the M.I.T. data to show that data from the three laboratories agree reasonably well within experimental error. In addition to the data, predicted curves are shown for both the FENE-P and FENE-PM models, as described in Sec. II B. The steady state Trouton ratio for the FENE-P model can be found by simplifying Eqs. (17) and (18), and is given by

$$
\operatorname{Tr}_{\infty} \cong 2 L^{2}\left(\frac{\eta_{p}}{\eta_{0}}\right)\left[\left(1-\frac{3}{L^{2}}\right)-\frac{1}{2 \mathrm{De}}+O\left(\mathrm{De}^{-2}\right) \ldots\right],
$$

while for the FENE-PM model the Trouton ratio is approximately

$$
\operatorname{Tr}_{\infty} \cong 2 \frac{n k_{B} T b N_{m}}{\eta_{0}}\left[\left(1-\frac{3}{b N_{m}}\right)-\frac{1}{2 \mathrm{De}}\right],
$$

where $b N_{m} \equiv L^{2}$. The FENE-PM model thus predicts a steady state extensional viscosity that is a factor of $\sum_{i=1}^{N_{m}} i^{-(2+\tilde{\sigma})}$ smaller $\left(\approx 2.4711\right.$ for $N_{m}=200$ and $\tilde{\sigma}=-0.5$, corresponding to $h^{*}=0.25$ in the Zimm model). This factor is a consequence of some 
of the elastic stress in the FENE-PM dumbbell model being redistributed into less elastic (lower time constant) modes that do not stretch during uniaxial elongation [McKinley (1998)]. Both the FENE-P and FENE-PM models predict that $\operatorname{Tr}_{\infty}$ rapidly approaches a constant value as De increases. For De between 10 and 30, the FENE-PM asymptote nearly agrees with the data. The FENE-P model appears to describe an upper bound for $\operatorname{Tr}_{\infty}$, while the FENE-PM model gives a lower bound. At higher De, the experimental values of $\operatorname{Tr}_{\infty}$ decrease and fall below the predicted FENE-PM value. This "extension thinning" at high De may be a consequence of a stress-dependent drag on the chain [Gupta et al. (2000)], or of intermolecular interactions of the highly extended chains. However, the effect is unavoidably coupled with the physical limitations of the motors used in filament stretching rheometers, for the following reason: the maximum Hencky strain realized in a filament stretching device must decrease for all $\dot{\varepsilon}>\dot{\varepsilon}^{*}$, in accordance with Eq. (23b). For example, in the M.I.T. device, with $V_{\max }=3.0 \mathrm{~m} / \mathrm{s}$ at $\dot{\varepsilon}=9.0 \mathrm{~s}^{-1}$, the final Hencky strain is $\varepsilon \approx 4.5$, which is clearly below the value needed to reach the steady state plateau observed in Fig. 13. Calculating the predicted FENE-PM values of $\operatorname{Tr}_{\max }$ that correspond to these finite Hencky strains $\varepsilon_{\text {max }}$ rather than the true steady state value attained at infinite strain allows the extension thinning effect to be qualitatively captured, as shown in Fig. 16. Although the Monash and Toronto devices achieved higher final Hencky strains than those at M.I.T., extension thinning was still observed. The approach to "true", steady state conditions may be very slow, so the constraints imposed by physical hardware limitations must be considered when analyzing data at higher strains and strain rates.

\section{DISCUSSION AND CONCLUSIONS}

The outcome of our collaborative project is a comprehensive characterization of both the shear and extensional rheology of a set of carefully prepared test fluids. In our molecular characterization of the solutions, intrinsic viscosity measurements yield an excluded volume exponent of $\nu=0.52$, indicating slightly better than theta solvent behavior. A Zimm bead-spring model predicts the linear viscoelasticity data remarkably well for all three fluids, and the resulting viscometric properties - the relaxation time $\lambda_{Z}$ and polymeric viscosity $\eta_{p}$-also scale with molecular weight in the manner expected. The excluded volume scaling exponents $\nu$ determined from both intrinsic viscosity and viscometric data agree remarkably well, to well within the estimated experimental error. FENE-P and FENE-PM model predictions are found to agree only qualitatively with steady and transient shear flow data. The FENE-PM model is slightly more successful than the FENE-P model at predicting some of the observed nonlinearities in the transient stress growth and relaxation curves.

The main goal of this study is to compare in detail measurements from three different filament stretching rheometers, in part to assess the accuracy and reproducibility of results obtained from this new type of device, and in part to demonstrate the current state of research in extensional rheometry. The imposed and measured flow kinematics during stretching are found to agree quantitatively for experiments with similar Deborah numbers performed at each laboratory. By paying careful attention to detail in the filament stretching experiment (proper sample loading, correct axial alignment, accurate measurements of fluid properties and temperature, calibration of sensors), and by consistent postprocessing of the raw data, excellent interlaboratory agreement is obtained for measurements of the transient extensional viscosity of the test fluid SM1, a dilute solution of polystyrene of molecular weight $2.0 \times 10^{6} \mathrm{~g} / \mathrm{mol}$ dissolved in oligomeric styrene. This agreement is obtained despite differences in instrument configuration. 
The set of filament stretching experiments span the useful operating ranges of the instruments, shown in Fig. 6, at least for SM-1, and we have thus been able to directly quantify the practical limitations of the device. Gravitational sagging and elastic instabilities are found to limit the useful operating space for SM-2 and SM-3. That is, sagging occurs at low strain rates, and fingering occurs at high strain rates and high strains. Nonetheless, we show that filament stretching devices can provide a significant amount of reliable, quantitative information regarding tensile stress growth in viscoelastic fluids. For the least elastic fluid, SM-1, the transient uniaxial elongational viscosity is observed to approach steady state conditions, and predictions from the FENE-P and FENE-PM models for $\operatorname{Tr}_{\infty}$ are shown to bound the measured values for moderate Deborah numbers. The transient extensional viscosities predicted by the FENE-PM model do not agree as well with the experimental values, especially at moderate strains. This is most likely a consequence of the distribution of internal microscopic configurations in the polymer chains, which can be captured only by molecular models, such as bead-rod or bead-spring models, which retain internal degrees of freedom.

We show that filament stretching rheometry has matured into a reliable method of measuring the response of viscoelastic fluids, particularly dilute polymer solutions, to a nearly ideal uniaxial extensional deformation. The kinematics of the flow effectively isolate extensional effects from those of shearing. Thus filament stretching rheometry has the potential to provide useful information for the development of constitutive equations. The information can also help predict behavior in more complex flows such as those that contain both shearing and elongation. The authors hope that filament stretching rheometry will become a standard rheological technique, and that measurements of transient extensional viscosity will be regularly reported alongside data for steady and unsteady shear.

\section{ACKNOWLEDGMENTS}

The research performed at M.I.T. was supported by NASA under Grant Nos. NAG31385 and NCC3-610. S.L.A. thanks the Fannie and John Hertz Foundation for supporting her graduate research. S.J.M. was supported in part by the Director, Office of Energy Research, Office of Basic Energy Sciences, Materials Science Division of the U.S. Department of Energy under Contract No. DE-AC03-76SF00098. The work at the University of Toronto was supported by the Natural Sciences and Engineering Research Council of Canada. The research at Monash University was supported by a program grant from the Australian Research Council.

\section{References}

Anna, S. L., C. Rogers, and G. H. McKinley, "On controlling the kinematics of a filament stretching rheometer using a real-time active control mechanism,"' J. Non-Newtonian Fluid Mech. 87, 307-335 (1999).

Berg, S., R. Kröger, and H. J. Rath, "Measurement of extensional viscosity by stretching large liquid bridges in microgravity,"' J. Non-Newtonian Fluid Mech. 55, 307-319 (1994).

Bird, R. B., R. C. Armstrong, and O. Hassager, Dynamics of Polymeric Liquids, Volume 1: Fluid Mechanics, 2nd ed. (Wiley, New York, 1987a).

Bird, R. B., C. F. Curtiss, R. C. Armstrong, and O. Hassager, Dynamics of Polymeric Liquids, Volume 2: Kinetic Theory, 2nd ed. (Wiley, New York, 1987b).

Boger, D. V., "A highly elastic constant-viscosity fluid," J. Non-Newtonian Fluid Mech. 3, 87-91 (1977/78). Boger, D. V. and H. Nguyen, “A model viscoelastic fluid,” Polym. Eng. Sci. 18, 1037-1043 (1978).

Brandrup, J., E. H. Immergut, and E. A. Grulke, Polymer Handbook, 4th ed. (Wiley, New York, 1999), Vol. 1.

Byars, J. A. B., A. Öztekin, R. A. Brown, and G. H. McKinley, "Spiral instabilities in the flow of highly elastic fluids between rotating parallel disks," J. Fluid Mech. 271, 173-218 (1994). 
Crosby, A. J. and K. R. Shull, “Adhesive failure analysis of pressure-sensitive adhesives,’ J. Appl. Polym. Sci. 37, 3455-3472 (1999).

Dealy, J. M., "'Official nomenclature for material functions describing the response of a viscoelastic fluid to various shearing and extensional deformations,', J. Rheol. 39, 253-265 (1995).

Domann, R. and G. H. McKinley, "An experimental investigation of the extensional kinematics of polymer solutions in a filament stretching rheometer,' Lehrstuhl für Strömungsmechanik (Erlangen, Universität Erlangen-Nürnberg, 1998), Vol. 68.

Doyle, P. S. and E. S. G. Shaqfeh, 'Dynamic simulation of freely-draining, flexible bead-rod chains: Start-up of extensional and shear flow,', J. Non-Newtonian Fluid Mech. 76, 43-78 (1998).

Doyle, P., E. S. G. Shaqfeh, G. H. McKinley, and S. H. Spiegelberg, "Relaxation of dilute polymer solutions following extensional flow,', J. Non-Newtonian Fluid Mech. 76, 79-110 (1998).

Ferguson, J., B. Reilly, and N. Granville, "Extensional and adhesion characteristics of a pressure sensitive adhesive,', Polymer 38, 795-800 (1997).

Flory, P. J., Principles of Polymer Chemistry (Cornell University Press, Ithaca, 1953).

Gaudet, S., G. H. McKinley, and H. A. Stone, "Extensional deformation of Newtonian liquid bridges," Phys. Fluids 8, 2568-2579 (1996).

Graessley, W. W., "Polymer chain dimensions and the dependence of viscoelastic properties on concentration, molecular weight and solvent power,' Polymer 21, 258-262 (1980).

Gupta, R. K. and T. Sridhar, "Elongational rheometers," in Rheological Measurement, edited by A. A. Collyer and D. W. Clegg (Elsevier Applied Science, London, 1988), pp. 211-245.

Gupta, R. K., D. A. Nguyen, and T. Sridhar, "Extensional viscosity of dilute polystyrene solutions-Effect of concentration and molecular weight,' Phys. Fluids 12, 1296-1318 (2000).

Harrison, G. M., J. Remmelgas, and L. G. Leal, "The dynamics of ultradilute polymer solutions in transient flow: comparison of dumbbell-based theory and experiment,' J. Rheol. 42, 1039-1058 (1998).

Hassager, O., M. I. Kolte, and M. Renardy, "Failure and nonfailure of fluid filaments in extension,' J. Non-Newtonian Fluid Mech. 76, 137-152 (1998).

Hur, J. S., E. S. G. Shaqfeh, and R. G. Larson, "Brownian dynamics simulations of single DNA molecules in shear flow,'” J. Rheol. 44, 713-742 (2000).

Ide, Y. and J. L. White, "The spinnability of polymer fluid filaments,'” J. Appl. Polym. Sci. 20, 2511-2531 (1976).

Jackson, J. P., K. Walters, and R. W. Williams, "A rheometrical study of Boger fluids," J. Non-Newtonian Fluid Mech. 14, 173-188 (1984).

Jain, A., D. S. Shackleford, and K. W. Koelling, The 68th Society of Rheology Annual Meeting, Galveston, Texas, 1997.

James, D. F. and K. Walters, "A critical appraisal of available methods for the measurement of extensional properties of mobile systems,", in Techniques of Rheological Measurement, edited by A. A. Collyer (Elsevier, New York, 1994), pp. 33-53.

Kolte, M. I., H. K. Rasmussen, and O. Hassager, "Transient filament stretching rheometer II: Numerical simulation," Rheol. Acta 36, 285-302 (1997).

Kröger, R., S. Berg, A. Delgado, and H. J. Rath, "Stretching behavior of large polymeric and Newtonian liquid bridges in plateau simulation,'” J. Non-Newtonian Fluid Mech. 45, 385-400 (1992).

Kwan, T. C. B. and E. S. G. Shaqfeh, "Brownian dynamics simulations of the stress and molecular configuration of polymers in exponential and linearly-ramped shear flow,' J. Non-Newtonian Fluid Mech. 82, 139-165 (1998).

Larson, R. G., Constitutive Equations for Polymer Melts and Solutions (Butterworths, Boston, 1988).

Larson, R. G., H. Hu, D. E. Smith, and S. Chu, "Brownian dynamics simulations of a DNA molecule in an extensional flow field,' J. Rheol. 43, 267-304 (1999).

Lee, E. C., M. J. Solomon, and S. J. Muller, "Molecular orientation and deformation of polymer solutions under shear: A flow light scattering study,' Macromolecules 30, 7313-7321 (1997).

Li, L., R. G. Larson, and T. Sridhar, "Brownian dynamics simulation in dilute polystyrene solutions,” J. Rheol. 44, 291-322 (2000).

MacDonald, M. J. and S. J. Muller, "Shear rheology of polymer solutions near the critical condition for elastic instability,', Rheol. Acta 36, 97-109 (1997).

Magda, J. J. and R. G. Larson, "A transition occurring in ideal elastic liquids during shear flow,' J. NonNewtonian Fluid Mech. 30, 1-19 (1988).

Matta, J. E. and R. P. Tytus, “Liquid stretching using a falling cylinder,' J. Non-Newtonian Fluid Mech. 35, 215-229 (1990).

McKinley, G. H., "Extensional flow and instabilities of elastic polymer solutions,"' in Dynamics of Complex Fluids: Proceedings of the 2nd Royal Society/Unilever Indo-UK Forum, edited by M. J. Adams, R. A. Mashelkar, J. R. A. Pearson, and A. R. Rennie (Imperial College Press, London, 1998).

McKinley, G. H., J. A. Byars, R. A. Brown, and R. C. Armstrong, "'Observations on the elastic instability in cone-plate and parallel plate flows of a polyisobutylene Boger fluid,' J. Non-Newtonian Fluid Mech. 40, 201-231 (1991). 
Münstedt, H. and H. M. Laun, “Elongational behavior of a low density polyethylene melt II. Transient behaviour in constant stretching and tensile creep experiments. Comparison with shear data. Temperature dependence of the elongational properties,', Rheol. Acta 18, 492-504 (1979).

Olagunju, D. O., "A 1-D theory for extensional deformation of a viscoelastic filament under exponential stretching," J. Non-Newtonian Fluid Mech. 87, 27-46 (1999).

Ooi, Y. W. and T. Sridhar, "Extensional rheometry of fluid S1,' J. Non-Newtonian Fluid Mech. 52 $153-162$ (1993).

Ooi, Y. W. and T. Sridhar, "Uniaxial extension of a lyotropic liquid crystalline polymer solution,'” IEC Res. 33, 2368-2373 (1994).

Orr, N. and T. Sridhar, "Stress relaxation in uniaxial extension,' J. Non-Newtonian Fluid Mech. 67, 77-104 (1996).

Orr, N. V. and T. Sridhar, "'Probing the dynamics of polymer solutions in extensional flow using step strain rate experiments,' J. Non-Newtonian Fluid Mech. 82, 203-232 (1999).

Petrie, C. J. S., Elongational Flows (Pitman, London, 1979).

Rasmussen, H. K. and O. Hassager, "Three-dimensional simulations of viscoelastic instability in polymeric filaments,' J. Non-Newtonian Fluid Mech. 82, 189-202 (1999).

Remmelgas, J., L. G. Leal, N. V. Orr, and T. Sridhar, "Viscous and elastic stresses in extensional rheometry,' J. Non-Newtonian Fluid Mech. 76, 111-135 (1998).

Shipman, R. W. G., M. M. Denn, and R. Keunings, "Mechanics of the 'falling plate' extensional rheometer,' J. Non-Newtonian Fluid Mech. 40, 281-288 (1991).

Sizaire, R. and V. Legat, "Finite element simulation of a filament stretching extensional rheometer," J. NonNewtonian Fluid Mech. 71, 89-107 (1997).

Solomon, M. J. and S. J. Muller, "Study of mixed solvent quality in a polystyrene-dioctyl phthalate-polystyrene system,’’ J. Polym. Sci., Part B: Polym. Phys. 34, 181-192 (1996a).

Solomon, M. J. and S. J. Muller, "The transient extensional behavior of polystyrene-based Boger fluids of varying solvent quality and molecular weight,' J. Rheol. 40, 837-856 (1996b).

Spiegelberg, S. H. and G. H. McKinley, "Stress relaxation and elastic decohesion of viscoelastic polymer solutions in extensional flow,' J. Non-Newtonian Fluid Mech. 67, 49-76 (1996).

Spiegelberg, S. H., D. C. Ables, and G. H. McKinley, "The role of end-effects on measurements of extensional viscosity in viscoelastic polymer solutions with a filament stretching rheometer,' J. Non-Newtonian Fluid Mech. 64, 229-267 (1996).

Sridhar, T., “An overview of the project M1,' J. Non-Newtonian Fluid Mech. 35, 85-92 (1990).

Sridhar, T., D. A. Nguyen, and G. G. Fuller, "Birefringence and stress growth in uniaxial extension of polymer solutions,'” J. Non-Newtonian Fluid Mech. 90, 299-315 (2000).

Sridhar, T., V. Tirtaatmadja, D. A. Nguyen, and R. K. Gupta, "Measurement of extensional viscosity of polymer solutions,' J. Non-Newtonian Fluid Mech. 40, 271-280 (1991).

Szabo, P., "Transient filament stretching rheometer I: Force balance analysis," Rheol. Acta 36, 277-284 (1997).

Tirtaatmadja, T. and T. Sridhar, "A filament stretching device for measurement of extensional viscosity,' J. Rheol. 37, 1081-1102 (1993).

Tirtaatmadja, V. and T. Sridhar, "Comparison of constitutive models for polymer solutions in uniaxial extension,', J. Rheol. 39, 1133-1160 (1995).

Trouton, F. T., "On the coefficient of viscous traction and its relation to that of viscosity," Proc. R. Soc. London 77, 426-440 (1906).

van Nieuwkoop, J. and M. M. O. Muller von Czernicki, “Elongation and subsequent relaxation measurements on dilute polyisobutylene solutions,'” J. Non-Newtonian Fluid Mech. 67, 105-124 (1996).

Verhoef, M. R. J., B. H. A. A. van den Brule, and M. A. Hulsen, “'On the modeling of a PIB/PB Boger fluid in extensional flow,' J. Non-Newtonian Fluid Mech. 80, 155-182 (1999).

Wedgewood, L. E., D. N. Ostrov, and R. B. Bird, "A finitely extensible bead-spring chain model for dilute polymer solutions," J. Non-Newtonian Fluid Mech. 40, 119-139 (1991).

Yao, M. and G. H. McKinley, "Numerical simulation of extensional deformations of viscoelastic liquid bridges in filament stretching devices," J. Non-Newtonian Fluid Mech. 74, 47-88 (1998).

Yao, M., S. H. Spiegelberg, and G. H. McKinley, "Dynamics of weakly strain-hardening fluids in filament stretching devices,' J. Non-Newtonian Fluid Mech. 89, 1-43 (2000). 